$$
\begin{array}{r}
\dot{r}^{2}+\left[\alpha^{2}(r, t) / r\right]\left[1+\frac{Q_{0}}{Q(r, t)}([r / a]-1) H([r / a]-1)\right] \\
=\alpha^{2}(r, 0) / s
\end{array}
$$

The charge contained within the surface of the sphere of radius $r$, at the time $t$ is $Q(r, t)$. It is related to the charge density $q$, through

$$
Q(r, t)=\int_{0}^{r} q(z, t) 4 \pi z^{2} d z
$$

In order to construct the function $q(r, t)$, we calculate the charge in the volume element $4 \pi r^{2} d r$. Suppose only two shells with $s$ numbers $s_{1}$ and $s_{2}$ intersect at the time $t$. Then the charge in the said volume element is

$$
\begin{aligned}
& 4 \pi r^{2} d r q(r, t)=4 \pi s_{1}^{2} d s_{1} q\left(s_{1}\right) \\
& \quad+4 \pi s_{2}^{2} d s_{2} q\left(s_{2}\right)-4 \pi r^{2} a^{2} d r Q_{0} \delta(r-a)
\end{aligned}
$$

or equivalently,

$$
\begin{aligned}
r^{2} q(r, t) & =\left[s^{2} \partial s / \partial r q_{0}(s)\right]_{s,} \\
& +\left[s^{2} \partial s / \partial r q_{0}(s)\right]_{s_{3}}-Q_{0} a^{2} \delta(r-a) .
\end{aligned}
$$

The initial charge distribution is $q_{0}$. The values $s_{1}$ and $s_{2}$ are the roots of the equation

$$
r-r(s, t)=0 \text {. }
$$

More generally, if this equation has, say, $N$ roots then Eq. (31) appears as

$q(r, t)=\sum_{i=1}^{n}\left(s_{l} / r\right)^{2}(\partial s / \partial r) s_{l} q\left(s_{l}\right)-Q_{0}(a / r)^{2} \delta(r-a)$.

Equations (28), (29), (32), and (33) are the desired equations of motion.

\section{ACRNOWLEDGMENTS}

The author is indebted to Dr. C. S. Gardner and Professor H. Weitzner for many helpful suggestions rendered during the early development of the paper. $\mathrm{He}$ is also grateful to S. Goldblatt of Nuclear Research Associates for suggesting relevant applications of these results, and feels especially indebted to Dr. John M. Dawson for his invaluable criticism of the final manuscript.

This research was supported in part by the Courant Institute of Mathematical Sciences, New York University, under Contract AT(30-1)-1480 with the U. S. Atomic Energy Commission; and in part by the Mechanics Branch of the Office of Naval Research under Contract Nonr 401(25) with the Graduate School of Aerospace Engineering, Cornell University.

\title{
The Most General Clebsch-Gordan Coefficients of the Universal Covering Group of the Inhomogeneous Lorentz Group*
}

\author{
Martin Kummer \\ The University of Michigan, Ann Arbor, Michigan
}

(Received 7 October 1964)

\begin{abstract}
The most general Clebsch-Gordan coefficients to reduce the physically most important $n$-fold product representations of the groups $\tilde{P}_{+}{ }^{\dagger}$ and $\tilde{P}$ (Universal covering groups of the restricted and full inhomogemeous Lorentz groups) are derived. They are used to answer the question: What can be said about the $S$-matrix if only Lorentz invariance is postulated?
\end{abstract}

\section{INTRODUCTION}

QEVERAL authors ${ }^{1-5}$ have constructed relativistic $D$ angular momentum states to achieve a general

* This work was supported by the National Science Foundation.

1 A. J. Macfarlane, Rev. Mod. Phys. 34, 41 (1962).

2 M. Jacob and G. C. Wick, Ann. Phys. 7, 404 (1959).

3 J. S. Lomont, J. Math. Phys. 1, 237 (1960).

4. Wehrle, Nucl. Phys. 44, 579, 637 (1963).

b H. Joos, Forschr. Physik 10, 65 (1962). "partial-wave decomposition" of the S-matrix. In this work, we intend to approach this problem from a quite general point of view. Namely, we want to determine the most general Clebsch-Gordan coefficients for an $n$-fold product of irreducible representations of $\tilde{P}_{+}^{\dagger}$ and $\tilde{P}$ (i.e., of the universal covering groups of the restricted and the full inhomogeneous Lorentz group), if only representations of $\tilde{P}_{+}^{\dagger}$ are considered with character $(m, \epsilon=+1, s)(m=$ 
mass, $\epsilon=\operatorname{sign}$ of the energy, $m \geq 0$ ), and if we confine ourselves to representations of $\tilde{P}$ with the same character, and for which, in addition, the square of the time-inversion and the square of the space-time inversion are represented by

$$
(-1)^{2 *} \text {. }
$$

For the explicit construction of these representations see Refs. 6-10.

We start from states which are represented by square-integrable functions of the four-momentum $p$ on the mass-hyperboloid $p^{2}=m^{2}$, and which at the same time are eigenfunctions of the spin operator

$$
S_{3}(p)
$$

[For its definition see (2.18).] It is well known that the spin operator of a particle in a coordinate system in which the four-momentum $p$ is measured is defined only up to a transformation induced by an element of the little group of $p$.

However, we want to start from states for which the spin of the $l$ th particle is defined quite generally. The only assumption we make is that the spin operators corresponding to physically indistinguishable particles are equally defined. For the convenience of the reader, we rederive many results which can already be found in literature at different places, e.g., the decomposition rules of the product representation of $\tilde{P}_{+}^{t}$ can be found in Refs. 3, 5, and 10 . The corresponding rules for $\tilde{P}$ which are also given in our paper, as far as the author knows, cannot be found elsewhere. The works of Jacob and Wick ${ }^{2}$ and Wehrle $^{4}$ served as a guide for the author. The present work may be looked upon as a generalization of their work, in which the helicity formalism is exclusively used.

We apply our methods also to the $S$-matrix which we first represent as a sum of covariants of $\tilde{P}_{+}^{\dagger}$ multiplied by invariant amplitudes. These may then be decomposed into partial-wave amplitudes. Finally, the connection between this approach and the $M$ functions of Stapp ${ }^{11,12}$ is given (see also Ref. 12).

\footnotetext{
- E. Wigner, Ann. Math. 40, No. 1 (1939).

7 V. Bargmann, Gruppentheoretische Analyse der Lorentzinvarianz, lecture given at the Federal Technical Institute, Zurich, 1963.

${ }^{8}$ V. Bargmann and E. Wigner, Proc. Natl. Acad. Sci. U.S. 34, (1948) 211.

${ }_{9}$ Fierz, Einführung in die Quantenfeldtheorie, lecture given at the Federal Technical Institute, Zurich 1963.

${ }_{10}$ A. S. Wightman in Relation de dispersion et particules elementaires $(1960)$, p. 161.

${ }_{11}$ H. P. Stapp, Phys. Rev. 125, 2139 (1962).

$1 \mathrm{~K}$. Hepp, Helv. Phys. Acta 37, 55 (1964).
}

\section{SOME FACTS ABOUT $S L(2, C)$}

It is well known that there exists a one-to-one correspondence between the Hermitian two-by-two matrices and the vectors of Minkowski space $p$,

$$
p=\left(p^{0}, p^{1}, p^{2}, p^{3}\right) \leftrightarrow p_{\sim}=\sigma^{\mu} p_{\mu},
$$

where

$$
\begin{aligned}
& \sigma^{0}=\left(\begin{array}{ll}
1 & 0 \\
0 & 1
\end{array}\right),=1, \quad \sigma^{1}=\left(\begin{array}{ll}
0 & 1 \\
1 & 0
\end{array}\right), \\
& \sigma^{2}=\left(\begin{array}{cc}
0 & -i \\
i & 0
\end{array}\right), \quad \text { and } \quad \sigma^{3}=\left(\begin{array}{cc}
1 & 0 \\
0 & -1
\end{array}\right)
\end{aligned}
$$

are the Pauli-matrices. It follows that

$$
\operatorname{det} p_{\sim}=\left(p^{0}\right)^{2}-p^{2}=m^{2} \text {. }
$$

We shall only consider the case where $p$ lies in the future cone.

By

$$
A p_{\sim} A^{\dagger}=[\Lambda(A) p]_{\sim},
$$

a two-one homomorphism between $S L(2, C)$ (Group of complex unimodular two-by-two matrices) and the restricted homogeneous Lorentz group is established. If we introduce the caret operation for any two-by-two matrix by the definition

$$
\hat{A}=\epsilon \bar{A} \epsilon^{-1}, \quad \epsilon=-i \sigma^{2}
$$

(the bar indicates the complex conjugate), it follows for $A \in S L(2, C)$

$$
\hat{A}=\left(A^{\dagger}\right)^{-1} \text {. }
$$

The caret operation is an outer automorphism of $S L(2, C)$, which, at the same time, is an involution. Because

$$
\hat{p}_{\sim}=[\Pi p]_{\sim},
$$

its application to (4) yields

$$
\hat{A}[\Pi p]_{\sim} \hat{A}^{\dagger}=[\Pi \Lambda(A) p]_{\sim},
$$

i.e.,

$$
\Pi \Lambda(A) \Pi=\Lambda(\hat{A}),
$$

where II denotes the space inversion. Note that the caret operation leaves the elements of $S U(2, C)$ (Group of the unitary unimodular matrices) invariant. Let $V^{+}(m)$ be the mass hyperboloid belonging to the mass $m$ in the future cone. The relation (4) then shows that to each $p \in V^{+}(m)$ there exists a transformation $\alpha(p) \in S L(2, C)$ with the property

$$
\begin{aligned}
m \alpha(p) \alpha^{\dagger}(p) & =p_{\sim} & & (m>0) ; \\
\frac{1}{2} \alpha(p)\left(\sigma_{0}+\sigma_{3}\right) \alpha^{\dagger}(p) & =p_{\sim} & & (m=0) .
\end{aligned}
$$

The sets $Q(p)$ of the transformations $\alpha(p)$, which possess these properties form left cosets of the little 
or isotropy groups of the matrices $\mathbb{1}(m>0)$ and $\frac{1}{2}\left(\sigma_{0}+\sigma_{3}\right)(m=0)$, respectively, which corresponds to the momentum $(m, 0)$ and $\left(\frac{1}{2}, 0,0, \frac{1}{2}\right)$ in Minkowski space. The isotropy groups are

$L(m)=\left\{\begin{array}{cc}S U(2, C) \subset S L(2, C), & m>0, \\ L(0) \quad \subseteq S L(2, C), & m=0,\end{array}\right.$

where $L(0)$ means the group of transformations

$$
\begin{gathered}
\tau(a) \rho(\varphi), \\
r(a)=\left(\begin{array}{ll}
1 & a \\
0 & 1
\end{array}\right), \\
\rho(\varphi)=\left(\begin{array}{cc}
e^{i \varphi / 2} & 0 \\
0 & e^{-i \varphi / 2}
\end{array}\right) .
\end{gathered}
$$

Specially important choices of the transformations $\alpha(p)$ are [we reserve parentheses for a general element in $Q(p)$, applying brackets and braces to the special elements quoted in the following]

$m>0$

$$
\alpha\{p\}=\left(\frac{p_{\sim}}{m}\right)^{\frac{1}{2}}=\frac{m+p_{\sim}}{[2 m(m+\omega(p))]^{\frac{1}{3}}}
$$

with

$$
\boldsymbol{\omega}(\mathfrak{p})=+\left(m^{2}+\mathfrak{p}^{2}\right)^{\frac{2}{2}},
$$

and, for $m \geq 0$,

$$
\alpha[p]=\rho[p] h[p] .
$$

Here

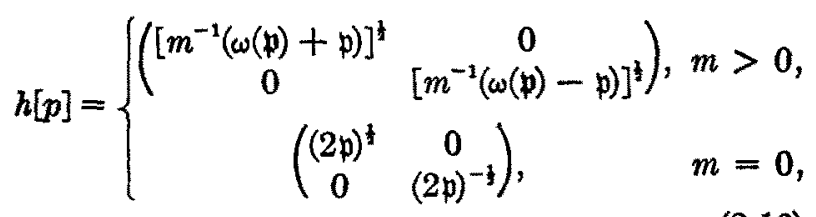

where $\mathfrak{p}$ is the Euclidian length of $\mathfrak{p}$,

and

$\rho[p]=\left\{\begin{array}{clc}e^{-i \varphi / 2 \sigma^{2}} e^{-i \vartheta / 2 \sigma^{2}} & \text { for }(0<\vartheta<\pi ; 0 \leq \varphi<2 \pi), \\ \mathbb{1} & \text { for } & \vartheta=0, \\ i \sigma^{1} & \text { for } & \vartheta=\pi .\end{array}\right.$

$\varphi, \vartheta$ are defined by

$$
p=p(\sin \vartheta \cos \varphi, \sin \vartheta \sin \varphi, \cos \vartheta) .
$$

In the case $m>0$ one has

$$
h[p]=\alpha\{p(3)\}
$$

with $p(3)=(\omega(\mathfrak{p}), 0,0, \mathfrak{p})$. In addition one derives from (14) for $v \in S U(2, C)$

$$
v \alpha\{p\} v^{\dagger}=\alpha\{\Lambda(v) p\} .
$$

From (19) and (20) we conclude that

$$
\alpha[p]=\rho[p] h[p]=\alpha\{p\} \rho[p] .
$$

\section{SURVEY ABOUT THE PHYSICALLY MOST IMPORTANT REPRESENTATIONS OF THE INHOMOGENEOUS LORENTZ GROUP1-‘}

Let $A=\left(\begin{array}{c}\alpha \beta \\ \gamma \delta\end{array}\right)$ be a matrix of $S L(2, C)$. It is well known that, by

$$
\begin{aligned}
& \frac{(\alpha x+\gamma y)^{s+\lambda}(\beta x+\delta y)^{*-\lambda}}{[(s+\lambda) !(s-\lambda) !]^{t}} \\
& \quad=\sum_{\lambda^{\prime}} \frac{x^{s+\lambda^{\prime}} y^{s-\lambda^{\prime}}}{\left[\left(s+\lambda^{\prime}\right) !\left(s-\lambda^{\prime}\right)\right]^{\frac{1}{2}}} D_{\lambda^{\prime} \lambda}^{*}(A)_{+},
\end{aligned}
$$

where $\lambda$ is one of the numbers of the set

$$
I_{+}(s)=\{-s,-s+1, \ldots 0,1 \cdots s-1, s\}
$$

and the summation over $\lambda^{\prime}$ is to be carried out over the same set, the representation $(s, 0)$

$$
A \rightarrow D^{\circ}(A)_{+}
$$

and the representation $(0, s)$

$$
A \rightarrow D^{*}(\hat{A})_{+}
$$

of $S L(2, C)$ is defined. If $A \in S U(2, C)$ the two representations coincide and become unitary.

Let $K$ be that subgroup of $S L(2, C)$, the elements of which are either of the form

$$
\left(\begin{array}{cc}
\alpha & 0 \\
0 & \alpha^{-1}
\end{array}\right)
$$

or of the form

$$
\left(\begin{array}{cc}
0 & \gamma^{-1} \\
\gamma & 0
\end{array}\right)
$$

Equation (1) then gives an irreducible representation of $K$ if we restrict $\lambda, \lambda^{\prime}$ to the set of the two numbers

$$
I_{0}(s)=\{-s, s\} .
$$

We denote the matrix which represents the element $A \in K$ in this representation by

$$
D^{\prime}(A)_{0} \text {. }
$$

We find

$$
D^{*}(A)_{0}= \begin{cases}\left(\begin{array}{cc}
\alpha^{2 *} & 0 \\
0 & \alpha^{-2 s}
\end{array}\right) & \text { for } A=\left(\begin{array}{cc}
\alpha & 0 \\
0 & \alpha^{-1}
\end{array}\right), \\
\left(\begin{array}{cc}
0 & (-\gamma)^{-2 t} \\
\gamma^{2 s} & 0
\end{array}\right) \text { for } A=\left(\begin{array}{cc}
0 & \gamma^{-1} \\
\gamma & 0
\end{array}\right) .\end{cases}
$$

We notice that the subgroup $K_{U}=K \cap S U(2, C)$ ( means intersection) is thus represented unitarily. $D^{*}(A)_{\tau}(\tau=0,+1)[A \in S L(2, C)$ for $\tau=+1$, 
$A \in K$ for $\tau=0$ ] defined in (1), and (2) is a matrix operating in a $N_{+}(s)$-dimensional vector space, where

$$
N_{\tau}(s)=\left\{\begin{array}{cl}
2 s+1, & \tau=+1, \\
2, & \tau=0 .
\end{array}\right.
$$

Let $\mathfrak{S}(m, s)$ be the Hilbert space of the matrices with one column and $N_{\tau}(s)$ rows, the elements of which are square-integrable functions defined on the mass-hyberboloid $V^{+}(m)$. We define the scalar product in $\mathfrak{S}(m, s)$ by

$$
(f, g)=\int_{V^{+}(m)} \frac{d^{3} p}{2 \omega(p)} f^{\dagger}(p) g(p) \quad f, g \in \mathfrak{E}(m, s),
$$

where the integration extends over $V^{+}(m)$ and the symbol ${ }^{\dagger}$ indicates the change to the adjoint (transposed and complex-conjugate) matrix.

The unitary irreducible representations of the orthochronous quantum mechanical Poincaré group $\tilde{P}^{\dagger}$, the universal covering group of the orthochronous inhomogeneous Lorentz-group $P^{\dagger}$ is defined in $\mathfrak{S}(m, s)$ by

$$
\begin{aligned}
(U(a) f)(p) & =e^{i p a} f(p), \\
(U(A) f)(p) & =D^{\prime}(\rho(p, A))_{r} f\left(\Lambda^{-1}(A) p\right), \\
(U(\Pi) f)(p) & =\eta_{\Pi} D^{\prime}\left(\rho_{\mathrm{I}}(p)\right)_{r} f(\Pi p) .
\end{aligned}
$$

The unimodular factor $\eta_{\mathrm{II}}$ is the parity. $\mathfrak{D}^{*}()_{\mathrm{r}}$ is defined in (1) and (2). [In future we drop the index $\tau$, keeping in mind that, in the case $m>0(m=0)$, we have to take the matrix $D^{\prime}()_{+}, D_{0}^{*}()$, respectively.]

The matrices $\rho$ are defined as follows

$$
\begin{aligned}
\rho(p, A) & =\alpha^{-1}(p) A \alpha\left(\Lambda^{-1}(A) \rho\right), \\
\rho_{\Pi}(p) & =\alpha^{\dagger}(p) \alpha(\Pi p),
\end{aligned}
$$

with arbitrary $\alpha(p) \in Q(p)$. [The physical significance of our choice of $\alpha(p)$ is seen below.] $\rho_{\text {II }}(p)$ has the properties

$$
\epsilon^{-1} \rho_{\Pi}(p) \in L(m), \quad \rho_{\Pi}(p) \rho_{\Pi}(\Pi p)=1 .
$$

Note that, for $m=0, \rho_{\Pi}(p) \notin L(0)$.

From (5e) one derives

$$
U^{2}(\Pi)=\eta_{\mathrm{a}}^{3} .
$$

These formulas are valid for cases $m>0$ and $m=0$, if one determines any matrix belonging to the group $L(0)$ modulus the invariant subgroup of "the translations" $\tau(a)$ as element of the group $D U(2, C)$ which is defined as the group of the matrices $\rho(\varphi)$ given in (2.13). [It is well known that only those representations of $L(0)$ have physical significance, in which the invariant subgroup of the "translations" is represented by the unity.]

By doing this, $\rho(p, A)$ as well as $\rho_{\mathrm{II}}(p)$ in the case $m=0$ become elements of $K_{U}$, so that $\mathfrak{D}^{\prime}()_{0}$ is defined for both of them. For $\alpha(p) \rightarrow \alpha[p]$, we get

$$
\epsilon^{-1} \rho_{\mathrm{a}}(p)=\left\{\begin{array}{c}
i \sigma^{3} \text { for }\left\{\begin{array}{l}
0 \leq \varphi<\pi \\
0<\vartheta<\pi
\end{array}\right\} \text { and } \vartheta=0, \\
-i \sigma^{3} \text { for }\left\{\begin{array}{l}
\pi \leq \varphi<2 \pi \\
0<\vartheta<\pi
\end{array}\right\} \text { and } \vartheta=\pi .
\end{array}\right.
$$

For $\alpha(p) \rightarrow \alpha\{p\}$, we get

$$
\rho_{\text {II }}\{p\}=1 \text {. }
$$

If we confine ourselves to the restricted group $\tilde{P}_{+}^{\dagger}$, the space $\mathfrak{S}(m, s)$ remains irreducible in the case $m>0$, decomposing into two subspaces with the elements

$$
\left(\begin{array}{c}
f_{+}(p) \\
0
\end{array}\right), \quad\left(\begin{array}{c}
0 \\
f_{-\bullet}(p)
\end{array}\right)
$$

respectively, in the case $m=0$.

Finally, we define the time-inversion antiunitary by

$$
\begin{aligned}
(V(T) f)(p) & =D^{*}(\epsilon)(\overline{U(\Pi) f})(p) \cdot \eta \\
& =D^{*}\left(\rho_{\mathrm{n}}(p) \epsilon\right) \vec{f}(\Pi p) \cdot \eta_{T}
\end{aligned}
$$

with

$$
\eta=\frac{\eta_{T}}{\eta_{\Pi}} \quad|\eta|^{2}=\left|\eta_{r}\right|^{2}=1 .
$$

Note that

$$
D_{\lambda^{\prime} \lambda}^{g}(\epsilon)=(-1)^{s-\lambda} \delta_{\lambda^{\prime},-\lambda}
$$

so that it follows

$$
V^{2}(T)=(-1)^{2 s} .
$$

Defining $V(T)$ by (12), it is not necessary to enlarge the space to represent the time inversion. We do not consider representations with different values of $U^{2}(I)$ and $V^{2}(T)$. These are found, for example, in Ref. 7.

If we define $V(\Pi T)$ by

$$
V(\Pi T)=U(\Pi) V(T)=\frac{\eta_{\Pi}}{\dot{\eta}_{\mathrm{n}}} V(T) U(\mathrm{I}),
$$

we get

$$
V^{2}(\mathrm{II} T)=(-1)^{2 t} \cdot \frac{\eta_{\mathrm{II}}}{\bar{\eta}_{\mathrm{II}}}
$$

The freedom in the choice of $\alpha(p) \in Q(p)$ is the mathematical expression of the fact that the spin vector of a particle in a coordinate system, in which 
the momentum $p$ is measured is only determined up to a transformation with an element out of the isotropy group of $p$. If $\mathfrak{\Im}$ is the vector of the total angular momentum in our representation (vector of the Hermitian represented infinitesimal rotations), we get for the spin operator in a coordinate system in which the particle has the momentum $p$,

$$
\begin{aligned}
S_{k}(p) & =U(\alpha(p)) W_{k}(p(m)) U^{-1}(\alpha(p)) \\
& =W_{\nu}(p) \Lambda_{k}^{\nu}\left(\alpha^{-1}(p)\right)
\end{aligned}
$$

Here

$$
\begin{aligned}
p(m) & =(m, 0) \text { for } m>0, \\
p(0) & =\left(\frac{1}{2}, 0,0, \frac{1}{2}\right), \\
W_{v}(p) & =\left[p^{0}(m)\right]^{-1}\left(\mathfrak{p} \cdot \mathfrak{J}, p^{0} \mathfrak{I}+\mathfrak{p} \times \mathfrak{l}\right),
\end{aligned}
$$

and $\mathfrak{N}$ is the vector of the Hermitian Operators representing the infinitesimal special Lorentz transformations in the three directions of our coordinatesystem.

For $\alpha(p) \rightarrow \alpha[p]$ we get

$$
\begin{aligned}
S_{3}[p] & =U(\alpha[p]) J_{3} U^{-1}(\alpha[p]) \\
& =U^{-1}(\rho[p]) J_{3} U^{-1}(\rho[p])=\mathfrak{J} \cdot \mathfrak{p} / \mathfrak{p},
\end{aligned}
$$

i.e., the projection of the total angular momentum on the direction of the momentum, the so-called helicity.

For $\alpha(p) \rightarrow \alpha\{p\}$ it follows

$$
\begin{aligned}
S_{3}\{\boldsymbol{p}\}= & \frac{1}{m}\left[-(\mathfrak{X} \cdot \mathfrak{p}) \frac{p_{3}}{m+\omega(\mathfrak{p})}\right. \\
& \left.+p_{0} J_{3}+p_{1} N_{2}-p_{2} N_{1}\right] \\
= & J_{3}-\frac{1}{m}\left(N_{1}-\frac{p_{2} J_{3}-p_{3} J_{2}}{m+\omega(\mathfrak{p})}\right) p_{2} \\
& +\frac{1}{m}\left(N_{2}-\frac{p_{3} J_{1}-p_{1} J_{3}}{m+\omega(\mathfrak{p})}\right) p_{1} \\
= & J_{3}-(\mathfrak{X} \times \mathfrak{p})_{3},
\end{aligned}
$$

where

$$
\mathfrak{X}=\frac{\mathfrak{N}}{m}-\frac{\mathfrak{p} \times \mathfrak{I}}{m(m+\omega(\mathfrak{p}))}+\mathfrak{p} f(\mathfrak{R}, \mathfrak{p})
$$

with suitable chosen $f(\mathfrak{\Re , p )}$ turns out to be the (Newton-Wigner) position operator.

Therefore, this choice of the transformation $\alpha(p)$ is equivalent to the definition of the spin (in a coordinate system in which the particle has momentum $p$ ) as difference between the total and the angular momentum.
Concluding this section, we mention that, in the case $m>0$, the transformation

$$
f^{\prime}(p)=\mathscr{D}^{s}(\alpha(p)) f(p)
$$

represents an isomorphism of the Hilbert space $\mathfrak{S}(m, s)$ and a Hilbert space equipped with the scalar product

$\left(f^{\prime}, g^{\prime}\right)=\int \frac{d^{3} p}{2 \omega(\mathfrak{p})}{f^{\prime}}^{\dagger}(p) D^{s}\left(\frac{\hat{p}_{\sim}}{m}\right) g^{\prime}(p)=(f, g)$.

In this Hilbert space the unitary representation of $\tilde{P}_{+}^{\dagger}$ takes the well-known " $(s, 0)$-Spinorfield-form"

$$
\begin{aligned}
& \left(U(a) f^{\prime}\right)(p)=e^{i p a} f^{\prime}(p), \\
& \left(U(A) f^{\prime}\right)(p)=D^{s}(A) f^{\prime}\left(\Lambda^{-1}(A) p\right), \\
& \left(U(\Pi) f^{\prime}\right)(p)=D^{\prime}\left(\frac{p_{\sim}}{m}\right) f^{\prime}(\Pi p) \cdot \eta_{\mathbb{\Pi}}, \\
& \left(V(T) f^{\prime}\right)(p)=D^{\circ}(\epsilon) f^{\prime}(\Pi p) \cdot \eta_{T} .
\end{aligned}
$$

Equations (23)-(25) remain valid, of course, if a caret is put over each matrix occuring in $D^{\circ}()$ leading to the " $(0, s)$-Spinorfield form" of the unitary representation of $\tilde{P}_{+}^{\dagger}$. The space inversion maps the so-defined representation spaces of $\tilde{P}_{+}^{\dagger}$ onto each other.

\section{PREPARATIONS FOR THE DECOMPOSITION OF THE GENERAL PRODUCT REPRESENTATION OF $\tilde{\mathbf{P}}_{+}{ }^{\dagger}$}

Let us first consider the set $\boldsymbol{P}_{n}(\mathbf{m})\left(\mathbf{m}=\left(m_{1}, \cdots m_{n}\right)\right.$ $\left.|\mathrm{m}|=\sum_{i=1}^{n} m_{i}>0\right)$ of the oriented closed polygons in three-dimensional space, which have $n$ edges labeled with "weights" $m_{1} \cdots m_{n}$. Strictly given the set of "weights" $m_{1} \cdots m_{n}$ such a polygon is still characterized by $n$ vectors $\mathfrak{t}_{1} \ldots \mathfrak{t}_{n}$ which satisfy the subsidiary condition

$$
\sum_{i=1}^{n} \mathfrak{t}_{i}=0
$$

We define the total weight of a polygon by

$$
M=\sum_{i=1}^{n}\left(\mathfrak{f}_{i}^{2}+m_{i}\right)^{\frac{1}{2}} .
$$

Instead of characterizing the polygon by $\mathbf{m}$ and $\left(\mathfrak{t}_{1} \ldots \mathfrak{h}_{n}\right)$, we may also characterize it by $n$ fourvectors

$$
\mathbf{k}=\left(k_{1} \cdots k_{n}\right), \quad k_{i}=\left[\left(\mathfrak{t}_{i}^{2}+m_{i}^{2}\right)^{\frac{1}{2}}, \mathfrak{t}_{i}\right],
$$

which fulfill the subsidiary condition

$$
\sum_{i=1}^{n} k_{i}=(M, 0)
$$


or applying the mapping (2.1) by $n$ Hermitian matrices

$$
\mathbf{k}_{\sim}=\left(k_{\sim 1} \cdots k_{\sim n}\right)
$$

satisfying the subsidiary condition

$$
\sum_{i=1}^{n} k_{\sim i}=M \cdot \mathbb{1} \text {. }
$$

Consequently, the weights of the edges are then given by the roots of the determinants of these matrices.

We call equivalent two of the elements of $\Phi_{n}(\mathrm{~m})$ if and only if there exists a rotation $R_{\varphi \vartheta \times}(\varphi, \vartheta, x=$ Euler angles) which transforms one of the elements into the other. Hence $\mathcal{O}_{n}(\mathrm{~m})$ decomposes into equivalence classes. Let us imagine that a system of representatives of these classes is chosen. Assume that it is given by polygons characterized by the vectors

$$
\mathfrak{b}_{i}=\mathfrak{b}_{i}(\boldsymbol{x}) \quad(i=1 \cdots n), \quad \sum_{i=1}^{n} \mathfrak{b}_{i}=0
$$

where $x=\left(\kappa_{1} \cdots \kappa_{3 n-8}\right)$ are $(3 n-6)$ inner (i.e., rotation-invariant) parameters which parametrize the system of representatives. We may choose as $\kappa_{1}$ (the square of) the total weight $M$. In general, $M$ is a function of $\boldsymbol{k}$ :

$$
M=M(\boldsymbol{x}) \text {. }
$$

A possible choice of the parameters is

$$
\mathfrak{b}_{1} \mathfrak{h}_{2} \cdots \mathfrak{b}_{n-1}, \quad \vartheta_{2}, \vartheta_{3} \varphi_{3} \cdots \vartheta_{n-1} \varphi_{n-1},
$$

where $\varphi_{k}, \vartheta_{k}$ are the polar angles of $\mathfrak{b}_{k}$ relative to $\mathfrak{b}_{1}(\varphi$ is measured starting from the plane put up by $\mathfrak{b}_{1}$ and $\mathfrak{b}_{2} ; \mathfrak{b}_{i}$ denotes the length of $\left.\mathfrak{b}_{i}\right)$.

It is possible to choose the Lorentz-invariant parametrization

$$
\begin{gathered}
\left(b, b_{1}\right) \cdots\left(b, b_{n-1}\right), \quad\left(b_{1}, b_{2}\right) \cdots\left(b_{1}, b_{n-1}\right), \\
{\left[b_{1}, b_{2}, b_{3}, b_{4}\right] \cdots\left[b_{n-3}, b_{n-2}, b_{n-1}, b_{n}\right],}
\end{gathered}
$$

where

$$
b_{k}=\left(\left[m_{k}^{2}+\mathfrak{b}_{k}^{2}\right]^{\frac{1}{2}}, \mathfrak{b}_{k}\right), \quad b=\sum_{i=1}^{n} b_{i},
$$

$\left(b_{1}, b_{2}\right)$ is the Minkowski scalar product of the vectors $b_{1}$ and $b_{2}$, and

$$
\left[b_{1}, b_{2}, b_{3}, b_{4}\right]=\epsilon^{\nu \mu \sigma \sigma} b_{1 \nu} b_{2 \mu} b_{3 \tau} b_{4 \sigma}
$$

stands for the volume in Minkowski space defined by the vectors $b_{1} \cdots b_{4}$.

In future, we use a general parametrization if we do not explicitly refer to a special one. The domain in $\kappa$-space, the points of which correspond to real polygons, we denote by $C_{n}$, and the submanifold of $C_{n}$ defined by (4.8) we denote by $C_{n}(M)$.

In the case $n=2, C_{n}$ is the positive real axis. One has

$$
\mathfrak{b}_{1}=-\mathfrak{b}_{2}=\mathfrak{b}
$$

and one may choose as inner parameter $\mathfrak{b}$ or $M$. The two quantities are connected by

$$
M=\left(\mathfrak{b}^{2}+m_{1}^{2}\right)^{\frac{1}{2}}+\left(\mathfrak{b}^{2}+m_{2}^{2}\right)^{\frac{1}{2}},
$$

i.e.,

$$
\mathfrak{b}=\Delta^{\frac{1}{2}}\left(m_{1}, m_{2}, M\right),
$$

where

$$
\begin{array}{r}
\Delta\left(m_{1}, m_{2}, M\right)=\left(1 / 4 M^{2}\right)\left(m_{1}^{4}+m_{2}^{4}+M^{4}\right. \\
\left.-2 m_{1}^{2} M^{2}-2 m_{2}^{2} M^{2}-2 m_{1}^{2} m_{2}^{2}\right) .
\end{array}
$$

It is no loss of generality to assume that $\mathfrak{b}_{1}$ points in the direction of the 3-axis in three-dimensional space, so that

$$
\begin{aligned}
& b_{\sim 1}=\left(\begin{array}{cc}
\omega_{1}(\mathfrak{b})+\mathfrak{b} & 0 \\
0 & \omega_{1}(\mathfrak{b})-\mathfrak{b}
\end{array}\right), \\
& b_{\sim 2}=\left(\begin{array}{cc}
\omega_{2}(\mathfrak{b})-\mathfrak{b} & 0 \\
0 & \omega_{2}(\mathfrak{b})+\mathfrak{b}
\end{array}\right) .
\end{aligned}
$$

We now want to parametrize one of the equivalenceclasses in $\mathcal{P}_{n}(\mathrm{~m})$. It is evident that, in the case $n \geq 3$, the three-dimensional rotation group (and in the case $n=2$, the two-dimensional sphere) yields a parametrization. However, the parametrization adapted to our purposes is the following. We consider the isotropy-group $G_{n} \subset S L(2, C)$ of the $n$-vector of Hermitian two-by-two matrices

$$
b_{\sim}=\left(b_{\sim 1} \cdots b_{-1}\right) \text {. }
$$

We find

$$
G_{n}= \begin{cases}D U(2, C), & n=2, \\ \text { cyclic group of the } & \\ \text { two elements }(\mathbb{1},-\mathbb{1}), & n \geq 3 .\end{cases}
$$

Here we have assumed that $b_{\sim 1}, b_{\sim 2}$ (in the case $n=2$ ) have the form (4.15) so that $D U(2, C)$ is, as above, the group of the matrices $\rho(\varphi)$ defined in (2.13). We denote by

$$
S U(2, C) / G_{n}
$$

the manifold of the left cosets of the group $G_{n}$ with respect to the group $S U(2, C)$. In the case $n \geq 3, G_{n}$ is an invariant subgroup; hence (4.17) is a group (isomorphic to the three-dimensional rotation group).

A parametrization of the manifold (4.17) is now 
seen to be equivalent to one of an equivalence class of $P_{n}(\mathrm{~m})$, i.e., if $\{\rho\}$ is a system of representatives of the left cosets, $\{\rho\}$ yields a parametrization of an equivalence class of $P_{n}(\mathrm{~m})$. Let $\mathbf{p}=$ $\left(p_{1}, \cdots p_{n}\right)$ be an element of the product set

$$
V_{n}^{+}(\mathrm{m})=V^{+}\left(m_{1}\right) \otimes V^{+}\left(m_{2}\right) \otimes \cdots \otimes V^{+}\left(m_{n}\right),
$$

and let $q$ denote

$$
q=\sum_{i=1}^{n} p_{i}
$$

Then we have

$$
q^{2} \geq|\mathrm{m}|^{2}>0
$$

Further we assume

$$
\alpha(q) \in Q(q)
$$

By the relation

$$
\alpha^{-1}(q) \mathbf{p}_{\sim}\left(\alpha^{\dagger}(q)\right)^{-1}=\mathbf{k}_{\sim},
$$

we attach to each $p \in V^{+}(\mathrm{m})$ a polygon and therefore a representative $\rho \in\{\rho\}$ and a point $\boldsymbol{x} \in C_{n}\left(\left[q^{2}\right]^{\frac{1}{2}}\right)$. On the other hand, if $x \in C_{n}\left(\left[q^{2}\right]^{\frac{1}{3}}\right)$ and $\rho \in$ $S U(2, C) / G_{n}, q^{2} \geq|\mathrm{m}|^{2}$, and $\mathrm{b}(\mathbf{k})$ is a representative of a class in $\mathcal{P}_{n}(\mathrm{~m})$, we get a vector $\mathrm{p} \in V^{+}(\mathrm{m})$ by the relation

$$
\mathbf{p}_{\sim}=\alpha(q) \rho \mathrm{b}_{\sim}(\mathbf{k}) \rho^{\dagger} \alpha^{\dagger}(q) .
$$

In other words, there exists a parametrization of the manifold $V^{+}(\mathrm{m})$ by the one-to-one correspondence

$$
\mathbf{p} \leftrightarrow q, \rho, \mathbf{\kappa},
$$

where $q^{2} \geq|\mathbf{m}|^{2}>0, \rho \in S U(2, C) / G_{n}, x \in C_{n}\left(\left[q^{2}\right]^{\frac{1}{2}}\right)$. By $\kappa \in C_{n}\left(\left[q^{2}\right]^{\frac{1}{2}}\right)$, a equivalence class of polygons in $\mathcal{P}_{n}(\mathrm{~m})$ with total weight $\left[q^{2}\right]^{\frac{1}{3}}$ is fixed. We call it the equivalence-class $\boldsymbol{x}$. We consider now equivalence-classes, which are related to the given one:

(1) The equivalence class of the mirror polygons, which we denote by IIx. (Note that in the cases $n=2,3: \Pi \kappa=\kappa$.)

(2) Let $\gamma_{n}^{\prime}$ be the subgroup of the symmetric group $\gamma_{n}$ of $n$ elements which contains all the elements permutating those edges of a polygon in $\mathcal{P}_{n}(\mathrm{~m})$ which have the same "weights", so that $\mathcal{P}_{n}(\mathrm{~m})$ is mapped onto itself by each $\pi \in \gamma_{n}^{\prime}$. The permuted equivalence-class $\boldsymbol{x}$ we denote by $\pi \kappa$.

Because the polygon characterized by $\Pi b(x)$ must lie in the equivalence class $\Pi r$, and, similarly, the polygon characterized by $\pi \mathrm{b}(\boldsymbol{x})$ in the equivalence class $\pi \kappa$, there exists transformations $v_{\text {II }}(\boldsymbol{\kappa}), v_{\pi}(\boldsymbol{\kappa}) \in$
$S U(2, C)$ with the property

$$
\mathbf{b}_{\sim}(\Pi \mathbf{k})=v_{\Pi}(\boldsymbol{\kappa})[\Pi \mathbf{b}(\mathbf{x})]_{\sim} v_{\Pi}^{\dagger}(\boldsymbol{x})
$$

and

$$
\mathbf{b}_{\sim}(\pi \mathbf{x})=v_{\pi}(\mathbf{k})[\pi \mathrm{b}(\mathbf{x})]_{\sim} v_{*}^{\dagger}(\boldsymbol{\kappa}),
$$

respectively. They are only determined up to an element in $G_{n}$. Furthermore, because of

$$
\Pi(\Pi \kappa)=\kappa, \quad \pi_{1}\left(\pi_{2}\right) \kappa=\left(\pi_{1} \pi_{2}\right) \kappa,
$$

we have

$$
\begin{array}{ll}
\text { i.e., } & v_{\Pi}(\boldsymbol{\kappa}) v_{\Pi}(\Pi \boldsymbol{k})=\rho\left(\chi_{\Pi}\right) \in G_{n}, \\
& 0 \leq \chi_{\Pi}<4 \pi \text { for } n=2, \\
& \chi_{\Pi}=0,2 \pi \quad \text { for } n \geq 3,
\end{array}
$$

and

$$
v_{\pi_{3}}\left(\pi_{1} \boldsymbol{x}\right) v_{\pi_{1}}(\boldsymbol{k})=\rho\left(\chi_{\pi_{1}, \pi_{1}}\right) v_{\pi_{0} \pi_{1}}(\boldsymbol{k}),
$$

where

$$
\rho\left(\chi_{x_{2}, x_{3}}\right) \in G_{n} .
$$

Before continuing the general discussion, we give the most convenient choice of $\mathbf{b}(\mathbf{x}), v_{\pi}(\mathbf{x})$ and $v_{\Pi}(\boldsymbol{x})$. For $n=2,3$, we have $b(\Pi x)=b(x)$; hence we may choose $b$ to be real and

$$
v_{x}(\boldsymbol{x})=\epsilon \text { for all } \boldsymbol{x} \in C_{n} .
$$

For $n \geq 4$, we could in principle choose $v_{\Pi}(\kappa)=1$ by choosing $\Pi \mathrm{Ilb}(\boldsymbol{k})$ as representative of the class $\Pi_{\boldsymbol{k}}$. However, we shall see that the choice (29) is the most convenient one for all $n^{*}$. It corresponds to the choice $Y \mathrm{~b}(\boldsymbol{x})$ for the class $\Pi \boldsymbol{k}$, where $Y$ denotes the reflection about the $x z$-plane.

For $n=2$, one has

$$
\mathbf{b}(\pi \boldsymbol{k})=\mathbf{b}(\boldsymbol{k}) \text {, }
$$

where $b(\boldsymbol{x})$ is given by (15), so that we may set

$$
v_{\pi}(\boldsymbol{\kappa})=\epsilon \text {. }
$$

If $n \geq 3$, and if we disregard all cases where either

or

$$
\begin{aligned}
& \Pi \kappa=\kappa \\
& \pi \kappa=\Pi \kappa,
\end{aligned}
$$

by recognizing that the set of the points $\kappa \in C_{n}$ with one of the properties (32) has zero (Lebesgue) measure in $x$-space (we shall see the reason why we may neglect sets of zero measure in $x$-space later on), we may choose

$$
v_{\pi}(\boldsymbol{k})=\mathbb{1}
$$

* At least if one considers space and time inversion simultaneously. 
We now claim that, simultaneously with the correspondence (4.21), one has

$$
\begin{aligned}
\Lambda^{-1}(A) \mathrm{p} & \leftrightarrow \Lambda^{-1}(A) q, \rho^{-1}(q, A) \rho, \boldsymbol{\kappa}, \\
\Pi p & \leftrightarrow \Pi q, \rho_{\Pi}^{-1}(q) \rho v^{-1}(\boldsymbol{\kappa}), \Pi \kappa, \\
\pi \mathrm{p} & \leftrightarrow q, \rho v_{\boldsymbol{\tau}}^{-1}(\boldsymbol{\kappa}), \pi \boldsymbol{\kappa} .
\end{aligned}
$$

We show the validity of these correspondences in the case of the space-inversion and leave the proof of the other ones to the reader.

Application of the caret operation to Eq. (4.20) yields

$$
\hat{\mathbf{p}}_{\sim}=\hat{\alpha}(q) \rho \hat{\mathrm{b}}_{\sim}(\boldsymbol{\kappa}) \rho^{\dagger} \hat{\alpha}^{\dagger}(q),
$$

i.e.,

$$
\begin{aligned}
{[\Pi \mathbf{m}]_{\sim} } & =\hat{\alpha}(q) \rho v_{\Pi}^{\dagger}(\boldsymbol{k}) \mathbf{b} \sim(\Pi \mathbf{k}) v_{\Pi}(\mathbf{k}) \hat{\alpha}^{\dagger}(q) \\
& =\boldsymbol{\alpha}(\Pi q) \rho_{\Pi}^{-1}(q) \rho v_{\Pi}^{-1}(\boldsymbol{k}) \mathbf{b}_{\sim}(\Pi q) v_{\Pi}(\boldsymbol{k}) \rho_{\Pi}(q) \alpha^{\dagger}(\Pi q) .
\end{aligned}
$$

The last equation shows the statement $(4.34 \mathrm{~b})$.

We now define the transformation

$$
a(\mathbf{p})=\alpha(q) \rho \quad \text { if } \quad \mathbf{p} \leftrightarrow q, \rho, \boldsymbol{\kappa} .
$$

With the help of (34), one easily derives

$$
\begin{aligned}
a\left(\Lambda^{-1}(A) \mathrm{p}\right) & =A^{-1} a(\mathbf{p}), \\
a(\Pi \mathbf{p}) & =a(\mathbf{p}) v_{\Perp}^{-1}(\mathbf{k}), \\
a(\pi \mathbf{p}) & =a(\mathbf{p}) v_{\pi}^{-1}(\mathbf{x}) .
\end{aligned}
$$

Now, let $u\left(b_{l}\right)$ be an element of $L\left(m_{l}\right)$ which may depend on $b_{l}[L(m)$ was defined in (2.10)], and let

$$
\alpha_{l}\left(p_{l}\right) \in Q\left(p_{l}\right)
$$

$[Q(p)$ is defined after Eq. (2.9)]. Then we define

$$
\alpha_{l}^{u}\left(b_{l}\right)=\alpha_{l}\left(b_{l}\right) u\left(b_{l}\right) \in Q\left(b_{l}\right)
$$

and

$$
\begin{aligned}
r_{l}(\mathbf{p}) & =u^{-1}\left(b_{l}\right) \rho_{l}\left(a^{-1}(\mathbf{p}), \Lambda^{-1}[a(\mathbf{p})] p_{l}\right)[\rightarrow(3.6)] \\
& =\left[\alpha_{l}^{u}\left(b_{l}\right)\right]^{-1} a^{-1}(\mathbf{p}) \alpha_{l}\left(p_{l}\right) \in L\left(m_{l}\right) .
\end{aligned}
$$

[As always, we have to determine the transformations of $L(0)$ as diagonal matrices belonging to $D U(2, C)$, calculating modulus "the translations."]

By a change of the representative $\rho$ of the left coset of $G_{n}$,

$$
\rho \rightarrow \rho \rho(\chi)\left\{\begin{array}{rr}
0<\chi<4 \pi, & n=2, \\
\chi=2 \pi, & n \geq 3,
\end{array}\right.
$$

one is led to the substitutions

$$
\begin{aligned}
& a(\mathbf{p}) \rightarrow a(\mathbf{p}) \rho(\chi), \\
& r_{l}(\mathbf{p}) \rightarrow\left\{\begin{array}{cl}
y\left(b_{l}, \chi\right) r_{l}(\mathbf{p}), & n=2, \\
-r_{l}(\mathbf{p}), & n \geq 3,
\end{array}\right.
\end{aligned}
$$

where

$$
y\left(b_{l}, \chi\right)=\left[\alpha_{l}^{u}\left(b_{l}\right)\right]^{-1} \rho(-\chi) \alpha_{l}^{u}\left(b_{l}\right)=\hat{y}\left(b_{l}, \chi\right) .
$$

Furthermore, one easily derives

$$
\begin{aligned}
r_{l}\left(\Lambda^{-1}(A) \mathbf{p}\right) & =r_{l}(\mathbf{p}) \rho_{l}\left(p_{l}, A\right), \\
r_{l}(\Pi \mathbf{p}) & =w_{\Pi}^{l}(\mathbf{k}) r_{l}(\mathbf{p}) \rho_{\Pi}^{2}\left(p_{l}\right), \\
r_{l}(\pi \mathbf{p}) & =w_{\pi}^{l}(\mathbf{k}) r_{\pi l}(\mathbf{p}), \quad \pi \in \gamma_{n}^{\prime},
\end{aligned}
$$

where

$w_{\Pi}^{l}(\mathbf{k})=\left[\alpha_{l}^{u}\left(b_{l}^{\pi}\right)\right]^{-1} v_{\Pi}(\boldsymbol{k}) \hat{\alpha}_{l}^{u}\left(b_{l}\right), \quad b_{l}^{\Pi} \equiv b_{l}(\Pi \mathbf{k})$, $w_{\pi}^{l}(\kappa)=\left[\alpha_{l}^{u}\left(b_{l}^{\pi}\right)\right]^{-1} v_{\pi}(\kappa) \alpha_{l}^{u}\left(b_{x l}\right), \quad b_{l}^{\pi} \equiv b_{l}(\pi \kappa)$.

We give the proofs of $(41 \mathrm{~b}, \mathrm{c})$.

$$
\begin{aligned}
r_{l}(\Pi \mathbf{p}) & =\left[\alpha_{l}^{u}\left(b_{l}^{\Pi}\right)\right]^{-1} v_{\Pi}(\boldsymbol{k}) a^{-1}(\mathbf{p}) \alpha_{l}\left(\Pi p_{l}\right) \\
& =\left[\alpha_{l}^{u}\left(b_{l}^{\Pi}\right)\right]^{-1} v_{\Pi}(\boldsymbol{k}) \hat{\alpha}_{l}^{u}\left(b_{l}\right) \hat{r}_{l}(\Pi \mathbf{p}) \rho_{\Pi}^{l}\left(p_{l}\right) ; \\
r_{l}(\pi \mathrm{p}) & =\left[\alpha_{l}^{u}\left(b_{l}^{\pi}\right)\right]^{-1} v_{\pi}(\mathbf{k}) a^{-1}(\mathbf{p}) \alpha_{l}\left(p_{\pi l}\right) \\
& =\left[\alpha_{l}^{u}\left(b_{l}^{\pi}\right)\right]^{-1} v_{\pi}(\boldsymbol{k}) \alpha_{l}^{u}\left(b_{\pi l}\right) r_{\pi l}(\mathbf{p})
\end{aligned}
$$

if and only if

$$
\alpha_{l}(p)=\alpha_{\pi l}(p) \text { for } \pi \in \gamma_{n}^{\prime} .
$$

The validity of Eq. (4.41c) is founded on the assumption (4.43). Note that, in the case $m_{l}=0$, $w_{\mathrm{n}}^{l}(\mathbf{k}) \notin L(0)$, but $w_{\mathrm{n}}^{l}(\mathbf{k}) \epsilon \in L(0)$ because of $\mathrm{Eq}$. (3.8), so that $w_{n}^{l}(\boldsymbol{r}) \epsilon$ is to be determined as an element of $D U(2, C)$.

We now remark that, for arbitrary fixed $\alpha_{l}\left(p_{l}\right) \in$ $Q\left(p_{\imath}\right)$, we may choose $u\left(b_{l}\right) \in L(m)_{l}$ always in such a way that, for all $l$,

$$
\alpha_{l}^{u}\left(b_{l}\right)=\alpha\left[b_{l}\right],
$$

where $\alpha[p]$ is the transformation defined in Eq. (2.15). With this choice of $u\left(b_{l}\right)$ and the choices of $v_{\text {II }}, v_{\pi}$ as quoted in Eqs. (4.29), (4.31), and (4.33), we get

$$
\begin{gathered}
w_{\Pi \mathrm{I}}^{l}(\boldsymbol{k})=\alpha\left[Y b_{l}\right]^{-1} \epsilon \hat{\alpha}\left[b_{l}\right]= \pm \bar{\alpha}\left[b_{l}\right]^{-1} \epsilon \hat{\alpha}\left[b_{l}\right] \\
= \pm \epsilon\left\{\begin{array}{c}
- \text { sign, if } \mathfrak{b}_{l} \text { points in the direction of the } \\
\text { negative 3-axis, } \\
+ \text { sign otherwise. }
\end{array}\right.
\end{gathered}
$$

With the restrictions, we have already put on the choice of $b_{l}$ in the case $n \geq 3$, it is always possible to choose these vectors in such a way that no one points into the direction of the negative 3 -axis. However, this is not possible in the case $n=2$ in which we have chosen $\mathfrak{b}_{1}$ pointing in the direction of the positive 3 -axis and $\mathfrak{b}_{2}=-\mathfrak{b}_{1}$. That is why we get

$$
w_{\mathrm{n}}^{l}(\mathrm{k})= \begin{cases}-\epsilon & n=2, \quad l=2 \\ +\epsilon & \text { otherwise. }\end{cases}
$$


Furthermore, we get in the case $n=2$

$$
\begin{aligned}
w_{\mathbf{r}}^{l}(\boldsymbol{\kappa}) & =\alpha\left[b_{l}\right]^{-1} \epsilon \alpha\left[\Pi b_{l}\right]=\alpha\left[b_{l}\right]^{-1} \epsilon \hat{\alpha}\left[b_{l}\right] \rho_{\mathrm{II}}\left[b_{l}\right] \\
& =\alpha^{-1}\left[b_{l}\right] \bar{\alpha}\left[b_{l}\right] \epsilon \rho_{\mathrm{II}}\left[b_{l}\right] .
\end{aligned}
$$

According to Eqs. (2.16), (2.17), and (3.10), it follows that

$$
w_{\pi}^{l}(\boldsymbol{\kappa})=-i \sigma_{3}, \quad l=1,2,
$$

and (4.40) yields

$$
\begin{aligned}
& y\left(b_{1}, \chi\right)=\alpha^{-1}\left[b_{1}\right] \rho(-\chi) \alpha\left[b_{1}\right]=\rho(-\chi), \\
& y\left(b_{2}, \chi\right)=\left(i \sigma_{1}\right)^{-1} \rho(-\chi) i \sigma_{1}=\rho(\chi) .
\end{aligned}
$$

Finally, we get in the case $n \geq 3$ with the choice (33),

$$
w_{\pi}^{l}(\mathbf{x})=1
$$

This choice given by (4.29), (4.31), (4.33), and (4.44) corresponds to the construction of angular momentum states by Wick, Jacob, and Wehrle. We refer to it as the WJW choice.

We have not yet finished the necessary preparations for the decomposition of the direct product

$$
\begin{aligned}
\mathfrak{E}_{n}(\mathbf{m}, \mathbf{s}) & =\mathfrak{S}\left(m_{1}, s_{1}\right) \\
& \otimes \mathfrak{E}\left(m_{2}, s_{2}\right) \otimes \cdots \otimes \mathfrak{S}\left(m_{n}, s_{n}\right),
\end{aligned}
$$

$\mathbf{m}=\left(m_{1}, \cdots m_{n}\right) \mathbf{s}=\left(s_{1} \cdots s_{n}\right)$. We have to study the change of the relativistic invariant volume element on $V^{+}(m)$ if we introduce the parameters standing on the right side of (4.21). We start from the invariant element of volume in $p$-space and write

$$
d^{4 n} p=d^{4(n-1)} p d^{4} q=d^{4(n-1)} k d^{4} q ; \quad q=\sum_{k=1}^{n} p_{k},
$$

where $\mathbf{k}=\Lambda^{-1}(\alpha(p))$ p. From

$$
p^{0} d p^{0}=m d m
$$

it follows

$$
\begin{aligned}
\frac{d^{8 n} p}{\omega_{1}\left(\mathfrak{p}_{1}\right) \cdots \omega_{n}\left(\mathfrak{p}_{n}\right)} & m_{n} d m_{n}, \\
& =\frac{d^{8(n-1)} k}{\omega_{1}\left(\mathfrak{b}_{1}\right) \cdots \omega_{n-1}\left(\mathfrak{b}_{n-1}\right)} M \frac{d^{\mathbf{3}} q}{\omega(\mathfrak{q})} d M,
\end{aligned}
$$

where

$$
\omega(\mathfrak{p})=+\left[m^{2}+\mathfrak{p}^{2}\right]^{\mathfrak{t}},
$$

or, because of

$$
M=\sum_{i=1}^{n}\left[m_{i}^{2}+\mathfrak{b}_{i}^{2}\right]^{\frac{1}{t}},
$$

we get

$\frac{d^{3 n} p}{\omega_{1}\left(\mathfrak{p}_{1}\right) \cdots \omega_{n}\left(\mathfrak{p}_{n}\right)}=\frac{d^{3(n-1)} k}{\omega_{1}\left(\mathfrak{b}_{1}\right) \cdots \omega_{n-1}\left(\mathfrak{b}_{n-1}\right) \omega_{n}\left(\mathfrak{b}_{n}\right)} M \frac{d^{3} q}{\omega(\mathfrak{q})}$.
The invariant volume element of the unitary group $S U(2, C)$ is

$$
d \rho=\left[16 \pi^{2}\right]^{-1} d \cos \vartheta d \varphi d \chi .
$$

We have normed it in such a way that the volume of $S U(2, C)$ is one. It coincides (up to a factor) with the invariant volume element of the three-dimensional rotation group. We therefore get $(n \geq 3)$

$\frac{1}{2^{n}} \cdot \frac{d^{3 n} p}{\omega_{1}\left(\mathfrak{p}_{1}\right) \cdots \omega_{n}\left(\mathfrak{p}_{n}\right)}=A_{n}(\boldsymbol{k}) d \rho \frac{d^{3} q}{2 \omega(\mathfrak{q})} d^{3 n-6}$,

where $\omega(\mathfrak{q})=\left[\mathfrak{q}^{2}+M^{2}(\boldsymbol{x})\right]^{\frac{1}{2}}$ also depends on $\boldsymbol{x}$ and $A_{n}(\boldsymbol{x})$ stands for the expression

$$
A_{n}(\mathfrak{k})=\frac{1}{2^{n-1}} \frac{\partial^{3(n-1)} k}{\partial \rho \partial \kappa} \cdot \frac{M(\mathfrak{k})}{\omega_{1}\left(\mathfrak{h}_{1}\right) \cdots \omega_{n}\left(\mathfrak{h}_{n}\right)} .
$$

Note that $A_{n}(\boldsymbol{x})$ is not dependent on $\rho$ because $d^{3(n-1)} k$ is rotation-invariant, i.e., it is constant on a equivalence class of $P_{n}(m)$. If we now choose that parametrization of the equivalence classes of $\mathcal{P}_{n}(\mathrm{~m})$ which is given in (9) and which we denote by $\boldsymbol{\kappa}_{0}$, we find

$$
\begin{aligned}
d^{3(n-1)} k= & 16 \pi^{2} \prod_{i=1}^{n-1} \mathfrak{b}_{i}{ }^{2} d \cos \vartheta_{2} d \Omega\left(\mathbf{e}_{3}\right) \\
& \times \cdots d \Omega\left(\mathbf{e}_{n-1}\right) d \rho d \mathfrak{b}_{1} \cdots d \mathfrak{b}_{n-1}
\end{aligned}
$$

(Compare also Ref. 4), where $d \Omega(\mathrm{e})=d \cos \vartheta d \varphi$ is the element of volume of the sphere in threedimensional space. Hence, the result is

$$
A_{n}\left(\boldsymbol{x}_{0}\right)=\frac{\pi^{2}}{2^{n-5}} \prod_{i=1}^{n-1} \frac{\mathfrak{b}_{i}^{2}}{\omega_{i}\left(\mathfrak{b}_{i}\right)} M\left(\mathfrak{\kappa}_{0}\right) \cdot \frac{1}{\omega_{n}\left(\mathfrak{b}_{n}\right)},
$$

where we have to set

$$
\mathfrak{b}_{n}=-\sum_{i=1}^{n-1} \mathfrak{b}_{i} ; \quad M\left(\mathfrak{k}_{0}\right)=\sum_{i=1}^{n} \omega_{i}\left(\mathfrak{b}_{i}\right) .
$$

We notice that, by choosing this parametrization, $A_{n}(\boldsymbol{k})$ has the same value on the equivalence class of the mirror polygons as on the original one

$$
A_{n}\left(\Pi \kappa_{0}\right)=A_{n}\left(\kappa_{0}\right),
$$

and, on the permuted class, it has the value

$$
A_{n}\left(\pi \kappa_{0}\right)=\mathfrak{b}_{n}^{2} \mathfrak{b}_{\pi-1 n}^{-2} A_{n}\left(\kappa_{0}\right) .
$$

In the case $n=2$, we get

$$
\begin{aligned}
\frac{1}{4} \frac{d^{3} p_{1} d^{3} p_{2}}{\omega_{1}\left(\mathfrak{p}_{1}\right) \omega_{2}\left(\mathfrak{p}_{2}\right)} & =\frac{d^{3} q}{2 \omega(\mathfrak{q})} A_{2}(\mathfrak{b}) d \Omega(\mathbf{e}) d \mathfrak{b} \\
& =\frac{d^{3} q}{2 \omega(\mathfrak{q})} A_{2}(M) d \Omega(\mathbf{e}) d M
\end{aligned}
$$


with $A(|\mathfrak{b}|)$ and $A(M)$ given by

$$
\begin{gathered}
A_{2}(|\mathfrak{b}|)=\frac{\mathfrak{b}^{2}}{2 \omega_{1}(\mathfrak{b}) \omega_{2}(\mathfrak{b})} M(\mathfrak{b}), \\
A_{2}(M)=\left(\frac{d M}{d \mathfrak{b}}\right)^{-1} A_{2}(\mathfrak{b})=\frac{\mathfrak{b}}{2}
\end{gathered}
$$

The last equation is a consequence of

$$
\frac{d M}{d \mathfrak{b}}=\left(\frac{1}{\omega_{1}(\mathfrak{b})}+\frac{1}{\omega_{2}(\mathfrak{b})}\right) \mathfrak{b}=\frac{M}{\omega_{1}(\mathfrak{b}) \omega_{2}(\mathfrak{b})} \mathfrak{b} .
$$

In the case $n=2$, the mirror and the permuted equivalence class of polygons coincide with the original one, on which $A$ is a constant.

\section{DECOMPOSITION OF AN N-FOLD PRODUCT OF SPACES}

We now consider the space (4.49), where the assumption $|\mathrm{m}|=\sum_{i=1}^{n} m_{i}>0$ is made. The elements of $\mathfrak{S}_{n}(\mathbf{m}, \mathbf{s})$ are column matrices $f, g$, with $N_{r_{2}}\left(s_{1}\right)$. $N_{r}\left(s_{2}\right) \cdots N_{r_{n}}\left(s_{n}\right)\left(\tau_{k}=\operatorname{sgn} m_{k}\right)$ rows, which depend on $\mathbf{p}: f(\mathbf{p}), g(\mathbf{p})$, and the scalar produet is defined by

$$
(f, g)=\int_{V^{+}(\mathbf{m})} \frac{d^{3 n} p}{\omega_{1}\left(\mathfrak{p}_{1}\right) \cdots \omega_{n}\left(\mathfrak{p}_{n}\right)} \cdot \frac{1}{2^{n}} f^{\dagger}(\mathbf{p}) g(\mathbf{p}) .
$$

The representation of $\tilde{P}$ in $\mathfrak{S}_{n}(\mathrm{~m}, \mathrm{~s})$ is, according to (3.5) and (3.12), given by

$$
\begin{aligned}
(U(a) f)(\mathbf{p}) & =e^{i q a} f(\mathbf{p}), \\
(U(A) f)(\mathbf{p}) & =\mathfrak{D}^{\mathbf{s}}(\rho(\mathbf{p}, A)) f\left(\Lambda^{-1}(A) \mathbf{p}\right), \\
(U(\Pi) f)(\mathbf{p}) & =\mathfrak{D}^{\mathbf{s}}\left(\rho_{\Pi}(\mathbf{p})\right) f(\Pi \mathbf{p}) \cdot \mathbf{n}_{\Pi}, \\
(V(T) f)(\mathbf{p}) & =\mathbb{D}^{\mathrm{s}}(\epsilon)(\overline{(U(\Pi) f)}(\mathbf{p}) \cdot \mathbf{n},
\end{aligned}
$$

where $D^{\mathbf{s}}\left(\rho_{\mathrm{n}}(\mathbf{p})\right)$ is defined by

$\mathbb{D}^{\mathrm{s}}\left(\rho_{\mathrm{I}}(\mathrm{p})\right)=\mathbb{D}^{*_{1}}\left(\rho_{1 \mathrm{I}}\left(p_{1}\right)\right) \otimes \cdots \otimes \mathbb{D}^{\mathrm{n}_{\mathrm{n}}}\left(\rho_{n \mathrm{\Pi}}\left(p_{n}\right)\right)$

and $D^{\mathbf{s}}(\boldsymbol{\rho}(\mathbf{p}, A))$ in a corresponding way. $(\otimes$ means Kronecker product.) Moreover,

$$
\mathbf{n}_{\mathrm{II}}=\eta_{1 \Pi} \cdots \eta_{n \Pi}, \quad \boldsymbol{n}=\eta_{1} \cdots \eta_{n} .
$$

Now we define a representation of $\gamma_{n}^{\prime}$ in $\mathfrak{W}_{n}(\mathbf{m}, \mathbf{s})$, where $\gamma_{n}^{\prime}$ is that subgroup of the symmetric group of $n$ elements, which permutes equal particles among themselves by

$$
\left(U\left(\pi^{-1}\right) f\right)(\mathbf{p})=C\left(\pi^{-1}\right) f(\pi \mathbf{p}),
$$

or, more explicitly,

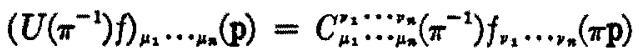

$$
\begin{aligned}
& =f_{\pi \mu_{2} \cdots \mu_{n}}(\pi \mathbf{p}) \text {. }
\end{aligned}
$$

By this equation the matrix $C$ is defined. The subspace of $\mathfrak{W}_{n}(\mathrm{~m}, \mathbf{s})$, which carries the physically correct representation of the group $\gamma_{n}^{\prime}$, we denote by $\mathfrak{S}_{n}^{\gamma}(\mathrm{m}, \mathrm{s})$. The elements belonging to this subspace satisfy the relation

$$
\left(U\left(\pi^{-1}\right) f\right)(\mathbf{p})=f(\mathbf{p}) \cdot \eta(\pi),
$$

where $\eta(\pi)$ is the signature of the fermion permutation under $\pi$. We now define

$$
D^{s}(\mathbf{p}) \equiv D^{s_{1}}\left(r_{1}(\mathbf{p})\right) \otimes \cdots \otimes D^{s_{n}}\left(r_{n}(\mathbf{p})\right)
$$

where $r_{l}(\mathbf{p})$ was defined in (4.38).

Moreover we define

$$
F(\mathbf{p})=D^{\mathbf{s}}(\mathbf{p}) f(\mathbf{p}) .
$$

Hence, we find according to (4.41)

$$
\begin{aligned}
& \left(U(A) F^{\prime}\right)(\mathbf{p})=D^{\mathbf{s}}(\mathbf{p})(U(A) f)(\mathbf{p})=F\left(\Lambda^{-1}(A) \mathbf{p}\right), \\
& \left(U(\Pi) F^{\prime}\right)(\mathrm{p})=D^{8}(\mathrm{p})(U(\Pi) f)(\mathrm{p}) \\
& =\mathfrak{D}^{\mathrm{s}}\left(w_{\mathrm{\Pi}}^{-1}(\mathrm{k})\right) F(\Pi \mathbf{p}) \cdot \mathfrak{n}_{\Pi}, \\
& \left(U\left(\pi^{-1}\right) F\right)(\mathbf{p})=\mathbf{D}^{\mathbf{s}}(\mathbf{p}) C\left(\pi^{-1}\right) f(\pi \mathbf{p}),
\end{aligned}
$$

or, because of

$$
D^{s}(\mathbf{p}) C\left(\pi^{-1}\right)=C\left(\pi^{-1}\right) D_{x}^{s}(\mathbf{p})
$$

[where

$$
\left.D_{x}^{s}(p)=D^{s x_{1}}\left(r_{\pi_{x}}(p)\right) \otimes \cdots \otimes D^{a x_{x}}\left(r_{x_{m}}(p)\right)\right]
$$

and $(4.41 \mathrm{c})$, we have

$$
\left(U\left(\pi^{-1}\right) F\right)(\mathrm{p})=C\left(\pi^{-1}\right) \mathbf{D}^{8}\left(w_{\pi}^{-1}(\mathrm{k})\right) F(\pi \mathrm{p}) .
$$

Besides the space $\mathfrak{\bigvee}_{n}(\mathrm{~m}, \mathrm{~s})$, we now consider the space of the column matrices $\tilde{\varphi}$ with $N_{r_{1}}\left(s_{1}\right), \ldots$ $N_{, n}\left(s_{n}\right)$ rows, which are square-integrable functions on the set

$$
\begin{gathered}
\{q, \rho, \boldsymbol{k}\} \quad q^{2} \geq|\mathbf{m}|^{2} ; \\
\rho \in S U(2, C) / G_{n} ; \quad \boldsymbol{x} \in C_{n}\left(\left[q^{2}\right]^{1}\right),
\end{gathered}
$$

and which, in addition, have the following properties [in the case $n=2$, we may drop the dependence of $\bar{\varphi}$ on $\kappa$ because $\kappa=\kappa_{1}$ is determined by $\left.M=\left(q^{2}\right)^{\frac{3}{3}}\right]$ :

$$
\begin{gathered}
n=2: \rho(\chi) \in D U(2, C), \\
\tilde{\varphi}(q, \rho \rho(\chi))=D^{\mathrm{s}}(y(\mathbf{b}, \chi)) \tilde{\varphi}(q, \rho),
\end{gathered}
$$

with $y\left(b_{l}, \chi\right)$ defined in (4.40);

for $n \geq 3$

$$
\tilde{\varphi}(q,-\rho, k)=(-1)^{2 l \cdot \mid} \tilde{\varphi}(q, \rho, k),
$$

where $|s|=\sum_{i=1}^{n} s_{i}$ and

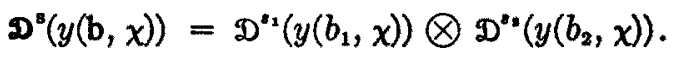


We denote the space of these functions by $\widetilde{\Re}_{n}(\mathbf{m}, \mathbf{s})$. The scalar product in it is defined by

$$
\begin{aligned}
& (\tilde{\varphi}, \bar{\psi})=\int d \rho \int d^{3 n-8}{ }_{\kappa} \\
& \times \int_{V^{+} M(\kappa)} \frac{d^{3} q}{2 \omega(\mathbf{q})} \tilde{\varphi}^{\dagger}(q, \rho, k) \psi(q, \rho, \boldsymbol{k}) \\
& =\int_{q^{2} \geq|\mathrm{m}|^{2}} d^{4} q \int d \rho \int d^{3_{n}-6} \kappa \theta(q) \delta\left(q^{2}-M^{2}(\boldsymbol{\kappa})\right) \\
& \times \tilde{\varphi}^{\dagger}(q, \rho, \kappa) \psi(q, \rho, \kappa),
\end{aligned}
$$

where

$$
\theta(q)= \begin{cases}1 & q_{0} \geq 0 \\ 0 & q_{0}<0\end{cases}
$$

It is invariant under the substitution

$$
\tilde{\varphi} \rightarrow D^{\mathfrak{s}}(y(\mathbf{b}, \chi)) \tilde{\varphi}
$$

because $y\left(b_{l}, \chi\right)$ is unitary.

According to (4.39), the definitions (5.8), and (3.1), the properties (5.12) and (5.13) are exactly the ones possessed by the functions $F(\mathbf{p})$, if they are comprehended as functions of $q, \rho$, and $x$. Hence, there exists a one-to-one correspondence between the elements of $\mathfrak{S}_{n}(\mathrm{~m}, \mathrm{~s})$ and that of $\tilde{\Omega}_{n}(\mathrm{~m}, \mathrm{~s})$. To make it an isomorphism between the Hilbert spaces,

$$
\mathfrak{S}_{n}(\mathrm{~m}, \mathrm{~s}) \cong \tilde{\mathfrak{R}}_{n}(\mathrm{~m}, \mathrm{~s}),
$$

we have to set

$$
\tilde{\varphi}(q, p, \boldsymbol{k})=A_{n}^{\frac{1}{2}}(\mathrm{k}) F(\mathrm{p}) .
$$

with $A_{n}(\mathrm{x})$ defined in (4.52) and (4.59). Indeed, we now get

$$
(\tilde{\varphi}, \tilde{\psi})=(F, g)=(f, g) .
$$

According to (4.34), Eq. (5.16) induces the following representation of $\tilde{P}$ and $\gamma_{n}^{\prime}$ in $\widetilde{\Re}_{n}(\mathbf{m}, \mathbf{s})$ :

$$
\begin{aligned}
& (U(a) \tilde{\varphi})(q, \rho, \kappa)=e^{i q a} \tilde{\varphi}(q, \rho, \boldsymbol{k}), \\
& (U(A) \tilde{\varphi})(q, \rho, \boldsymbol{\kappa})=\tilde{\varphi}\left(\Lambda^{-1}(A) q, \rho^{-1}(q, A) \rho, \boldsymbol{\kappa}\right), \\
& (U(\Pi) \vec{\varphi})(q, \rho, x)=n_{\Pi} B_{\Pi}(x) \\
& \times D^{s}\left(w_{\Pi}^{-1}(\kappa)\right) \tilde{\varphi}\left(\Pi q, \rho_{\Pi}^{-1}(q) \rho v_{\Pi}^{-1}(\kappa), \Pi \kappa\right), \\
& \left(U\left(\pi^{-1}\right) \tilde{\varphi}\right)(q, \rho, \kappa)=B_{\pi}(\boldsymbol{x}) C\left(\pi^{-1}\right) \\
& \times D^{s}\left(w_{x}^{-1}(\boldsymbol{x})\right) \vec{\varphi}\left(q, \rho v_{x}^{-1}(\boldsymbol{k}), \pi \boldsymbol{k}\right),
\end{aligned}
$$

where

$$
B_{\Pi}(\boldsymbol{k})=\left[\frac{A_{n}(\boldsymbol{k})}{A_{n}(\Pi \boldsymbol{k})}\right]^{\xi} ; \quad B_{\mathbf{r}}(\boldsymbol{k})=\left[\frac{A_{n}(\boldsymbol{k})}{A_{n}(\pi k)}\right]^{\xi} .
$$

If we now make the WJW choice of the adjustable quantities given in (4.29), (4.31), (4.33), (4.44) and assume a parametrization of the equivalence classes of $P_{n}(m)$ for which

$$
B_{\mathrm{I}}(\mathrm{x})=1
$$

[this condition is for example satisfied for the parametrization, given in (4.9)], we get

$$
\begin{aligned}
& (U(a) \tilde{\varphi})(q, \rho, \boldsymbol{k})=e^{i q a} \tilde{\varphi}(q, \rho, \boldsymbol{k}), \\
& (U(A) \tilde{\varphi})(q, \rho, \kappa)=\bar{\varphi}\left(\Lambda^{-1}(A) q, \rho^{-1}(q, A) \rho, k\right), \\
& (U(\Pi) \tilde{\varphi})_{2}(q, \rho, \kappa) \\
& =(-1)^{|s|-|\lambda|} \bar{\varphi}_{-2}\left(\Pi q, \rho_{\mathrm{II}}^{-1}(q) \rho \epsilon^{-1}, \Pi \kappa\right) \cdot n_{\Pi},
\end{aligned}
$$

where

$$
|s|=\sum_{i=1}^{n} s_{i} ; \quad|\lambda|= \begin{cases}\sum_{i=1}^{n} \lambda_{i}, & n \geq 3, \\ \lambda_{1}-\lambda_{2}, & n=2 ;\end{cases}
$$

and, for $n=2$,

$$
\left(U\left(\pi^{-1}\right) \ddot{\varphi}\right)_{\lambda_{1} \lambda_{z}}(q, \rho)=(-1)^{\lambda_{1}+\lambda_{2}} \ddot{\varphi}_{\lambda_{2} \lambda_{1}}\left(q, \rho \epsilon^{-1}\right),
$$

for $n \geq 3$,

$\left(U\left(\pi^{-1}\right) \tilde{\varphi}\right)(q, \rho, \boldsymbol{k})=B_{\pi}(\boldsymbol{k}) C\left(\pi^{-1}\right) \tilde{\varphi}(q, \rho, \pi \kappa) ; \pi \in \gamma_{n}^{\prime}$.

This means that the states belonging to the physically correct representation of the group have the property

$n \geq 3 \quad B_{\pi}(\boldsymbol{k}) C\left(\pi^{-1}\right) \tilde{\varphi}(q, \rho, \pi \boldsymbol{\kappa})=\eta(\pi) \tilde{\varphi}(q, \rho, \boldsymbol{\kappa})$.

In the case $n=2$, if the two particles are equal,

$$
\bar{\varphi}_{\lambda_{1} \lambda_{s}}(q, \rho)=\bar{\varphi}_{\lambda_{s} \lambda_{1}}\left(q, \rho \epsilon^{-1}\right)(-1)^{\lambda_{2}+\lambda_{2}} \cdot \eta(\pi) \text {. }
$$

Because of (4.47), the property (5.12) of $\varphi$ takes the form

$$
\tilde{\varphi}_{\lambda_{2} \lambda_{2}}(q, \rho \rho(\chi))=e^{-i\left(\lambda_{1}-\lambda_{z}\right) x} \bar{\varphi}_{\lambda_{2} \lambda_{2}}(q, \rho) .
$$

We now execute the transformation

$\dot{\varphi}(q ; J \lambda \sigma ; \boldsymbol{\kappa})=(2 J+1)^{\frac{1}{2}} \int d \rho \tilde{\varphi}(q, \rho, \boldsymbol{k}) D_{\lambda \sigma}^{J}(\rho)$,

which possesses the inverse

$\tilde{\varphi}(q, \rho, k)=\sum_{J, \sigma, \lambda}(2 J+1)^{\frac{1}{3}} \dot{\varphi}(q ; J \lambda \sigma ; \kappa) \bar{D}_{\lambda_{\sigma}}^{J}(\rho)$,

and which defines an isomorphism between $\widetilde{\Omega}_{n}(\mathrm{~m}, \mathrm{~s})$ and a new Hilbert space, with a scalar product defined as

$$
\begin{aligned}
& (\dot{\varphi}, \dot{\psi})=\sum_{\gamma \sigma \lambda} \int d^{3 n-6} \int_{V^{+} \mu(*)} \frac{d^{3} q}{2 \omega(\mathfrak{q})} \\
& \times \dot{\varphi}^{\dagger}(q ; J \lambda \sigma ; \boldsymbol{x}) \grave{\psi}(q ; J \lambda \sigma ; \boldsymbol{x}) \\
& =\sum_{J \sigma \lambda} \int_{C_{n}} d^{3 n-6} \kappa \int_{q^{2} \geq|m|^{2}} d^{4} q \theta(q) \delta\left(q^{2}-M^{2}(\mathrm{k})\right) \\
& \times \stackrel{\circ}{\varphi}^{\dagger}(q ; J \lambda \sigma ; \boldsymbol{k}) \stackrel{ }{\psi}(q ; J \lambda \sigma ; \boldsymbol{k}) .
\end{aligned}
$$


The transformed relations (5.13), (5.22), and (5.21) are

$n \geq 3: \stackrel{\circ}{ }(q ; J \lambda \sigma ; \kappa)\left[1-(-1)^{2(J-|\sigma|)}\right]=0$,

$n=2: \dot{\varphi}_{\lambda_{1} \lambda_{3}}(q ; J \lambda \sigma)\left(1-e^{i\left[\sigma-\left(\lambda_{2}-\lambda_{z}\right)\right] x}\right)=0$,

$\stackrel{\circ}{\varphi}(q ; J \lambda \sigma)=C(\pi) \stackrel{\varphi}{\varphi}(q ; J, \lambda,-\sigma)(-1)^{J-2 \varepsilon} \cdot \eta(\pi)$,

and the representation of $\tilde{P}_{+}^{+}$gets the demanded form in which it decomposes into irreducible parts,

$(U(a) \stackrel{\varphi}{\varphi})(q ; J \lambda \sigma ; \kappa)=e^{i q a} \stackrel{\varphi}{\varphi}(q ; J \lambda \sigma ; \kappa)$,

$(U(A) \stackrel{\bullet}{ })(q ; J \lambda \sigma ; \kappa)$

$$
=\mathbb{D}_{\lambda^{\prime}}^{J}(\rho(q, A)) \stackrel{\varphi}{\varphi}\left(\Lambda^{-1}(A) q ; J \lambda^{\prime} \sigma ; \boldsymbol{\kappa}\right),
$$

$(U(\mathrm{II}) \stackrel{\varphi}{\varphi})_{2}(q ; J \lambda \sigma ; \boldsymbol{\kappa})$

$=n_{\Pi}(-1)^{J-\sigma-|\rho|+|\lambda|} D_{\lambda \lambda^{\prime}}^{J}\left(\rho_{\Pi}(q)\right) \dot{\varphi}_{-2}\left(\Pi q ; J \lambda^{\prime},-\sigma ; \Pi \kappa\right)$,

$$
\begin{aligned}
& (V(T) \stackrel{\circ}{\varphi})(q ; J \lambda \sigma ; \boldsymbol{\kappa})
\end{aligned}
$$

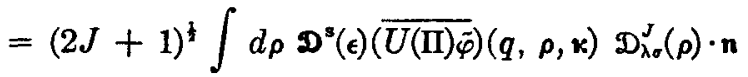

$$
\begin{aligned}
& =D_{\lambda \lambda}^{J} \cdot\left(\rho_{\Pi}(q) \epsilon\right) \bar{\varphi}\left(\Pi q ; J \lambda^{\prime} \sigma ; \Pi \boldsymbol{\kappa}\right) \cdot \mathbf{n}_{T} .
\end{aligned}
$$

If we denote by the symbol $\wp_{n}(x)$ the set of points in $x$-space on which the function

$$
\stackrel{\circ}{\varphi}_{\lambda}(q ; J \lambda \sigma ; \boldsymbol{\kappa})
$$

may be arbitrarily defined [defining $\varphi$ on the following sets is, of course, equivalent with defining $f_{2}(\mathbf{p})$ on the sets: $\left.\mathbf{p} \in V^{+}(\mathrm{m}) ; \lambda \in \Pi_{\mathrm{k}-1}^{n} \otimes I_{r k}\left(s_{k}\right)\right]-$ with the restriction that $(\dot{\varphi}, \dot{\varphi})$ is finite - we get according to (5.26) and (5.27)

$$
\begin{aligned}
& \grave{\vartheta}_{n}(q)=\left\{q ; q^{2} \geq|\mathbf{m}|^{2}\right\}, \\
& \wp_{n}(J)=\{J ; 2 J \equiv 2|s| \bmod 2\} \text {, } \\
& \wp_{n}(\sigma, \lambda)=\left\{\begin{array}{l}
I_{+}(J) \otimes \prod_{k=1}^{n} \otimes I_{r_{k}}\left(s_{k}\right) ; \quad n \geq 3, \\
{\left[\sigma=\lambda_{1}-\lambda_{2} ; \lambda \in I_{\tau_{1}}\left(s_{1}\right)\right.} \\
\left.\quad \otimes I_{\tau_{2}}\left(s_{2}\right) ;\left|\lambda_{1}-\lambda_{2}\right| \leq J\right] ; \quad n=2
\end{array}\right. \\
& \S_{\mathbf{u}}(\boldsymbol{x})=C_{n}\left[\left(q^{2}\right)^{\frac{1}{2}}\right] \text {. }
\end{aligned}
$$

The set $I_{r_{1}}\left(s_{1}\right) \otimes I_{r_{1}}\left(s_{2}\right)$ forms a rectangle $\left(s_{1} \neq s_{2}\right)$ or a square $\left(s_{1}=s_{2}\right)$ in the $\lambda_{1} \lambda_{2}$-plane.

$\grave{\vartheta}_{2}(\lambda)$ consists of those points of this set simultaneously lying between or on the straight lines given by the equations

$$
\lambda_{1}-\lambda_{2}=J, \quad \lambda_{2}-\lambda_{1}=J .
$$

[In the case $n=2$, we may drop the dependence of $\dot{\varphi}$ on $\boldsymbol{\kappa}$ and $\sigma$ because $\boldsymbol{\kappa}$ is determined by $M=$ $\left(q^{2}\right)^{\frac{1}{2}}$ and $\sigma$ by 2.$]$

The space of these functions $\dot{\varphi}$ equipped with the scalar product (5.25) we denote by $\hat{\Omega}_{n}(\mathrm{~m}, \mathrm{~s})$.

If two or more of the particles are equal, and if $\gamma_{n}^{\prime}$ is, as above, the group of those permutations which interchange equal particles among themselves, the functions $\stackrel{\circ}{\varphi}$, in addition, have the property

$B_{\pi}(\boldsymbol{k}) C\left(\pi^{-1}\right) \stackrel{\varphi}{\varphi}(q ; J \lambda \sigma ; \pi \boldsymbol{k})=\eta(\pi) \dot{\varphi}(q ; J \lambda \sigma ; \boldsymbol{k})$

for $n \geq 3$, and

$$
C\left(\pi^{-1}\right) \stackrel{\varphi}{\varphi}(q ; J \lambda)(-1)^{J-2 s} \cdot \eta(\pi)=\dot{\varphi}(q ; J \lambda)
$$

for $n=2$.

The space of these functions equipped with the scalar product (5.25) we denote by $\stackrel{\circ}{n}_{n}^{\gamma}(\mathrm{m}, \mathrm{s})$ and the set on which the function comprehended as a function of $x$ may be defined arbitrarily [up to the re-

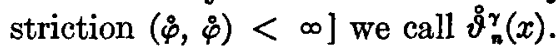

Let $\tau$ be a set of points in $\lambda$-space. We introduce a measure on these sets by the definition

$$
\mu(\tau)=\text { number of points contained in } \tau \text {. }
$$

We call a set $\tau_{1}$ smaller than a set $\tau_{2}$ if and only if

$$
\mu\left(\tau_{1}\right)<\mu\left(\tau_{2}\right) .
$$

We do the same in $x$-space except we take as measure the Lebesgue measure $\mu(\boldsymbol{k})$. $\vartheta_{n}^{\gamma}(\lambda)$ belongs to the set of the smallest subsets $\tau$ of $\vartheta_{n}(\lambda)$ which have the property

$$
\bigcup_{\pi \in \gamma_{n},} \pi \tau=\grave{\vartheta}_{n}(\lambda)
$$

(the symbol Umeans "union"). An analogous statement holds for $\xi_{n}(\boldsymbol{x})$.

In the case $n=2$, we only have to consider $\xi_{2}^{\gamma}(\lambda)$ which may be chosen as that subset of the square formed by $\vartheta_{2}(\lambda)$ lying on the upper side of the diagonal given by the equation $\lambda_{1}=\lambda_{2}$, the diagonal included or not depending on whether the upper or lower sign holds in the equation

$$
\eta(\pi)(-1)^{J-2 s}= \pm 1 \text {. }
$$

Comparing (5.29) with (3.5) and (3.12) we remark that the space $h_{n}(m, s)$, although reduced with respect to the group $\tilde{P}_{+}^{t}$, is still not reduced with respect to $\tilde{P}$. But, because

$$
V^{2}(T)=(-1)^{2 J},
$$

$V^{2}(\Pi T)=(-1)^{2 J} \quad$ (if all the $\eta_{\Pi}$ 's are real), 
it should be possible to perform an additional transformation in such a way that $\Pi$ and $T$ are represented in agreement with (3.5c) and (3.12) (see Ref. 7).

The transformation we are looking for is given by

$$
\begin{aligned}
\varphi(q, & \left.J \lambda ; \sigma \lambda_{x} \xi\right)=\frac{1}{4}\left\{\dot{\varphi}_{2}(q ; J \lambda \sigma ; \kappa)+\xi_{2} \dot{\varphi}_{2}(q ; J \lambda \sigma ; \Pi \kappa)\right. \\
& +\xi_{1} \xi_{2}(-1)^{J-\sigma-|\sigma|+|\lambda|}\left[\dot{\varphi}_{-2}(q ; J \lambda,-\sigma ; \kappa)\right. \\
& \left.\left.+\xi_{2} \dot{\varphi}_{-2}(q ; J \lambda,-\sigma ; \Pi \kappa)\right]\right\} .
\end{aligned}
$$

Here $\xi=\left(\xi_{1}, \xi_{2}\right)$ are two new parameters both of which, in general, can take the values $(+1,-1)$.

$\varphi$ has again the properties (5.26), (5.27), and, in addition, it satisfies the relations

$$
\begin{aligned}
& \varphi(q, J \lambda ; \sigma \lambda, \Pi \kappa, \xi)=\xi_{2} \varphi\left(q, J \lambda ; \sigma \lambda_{k} \xi\right), \\
& \varphi(q, J \lambda ;-\sigma,-\lambda \kappa \xi) \\
& \quad=\xi_{1} \xi_{2}(-1)^{J-\sigma-|\bullet|+|\lambda|} \varphi\left(q, J \lambda ; \sigma \lambda_{k} \xi\right) .
\end{aligned}
$$

By $\Omega_{n}(m, s)$, we denote the space of the functions $\varphi$ with all these properties equipped with the scalar product

$$
\begin{aligned}
(\varphi, \psi)= & \sum_{J \lambda} \sum_{\sigma \lambda \xi} \int d^{3 \alpha-0} \kappa \int_{\nabla+\mu(\kappa)} \frac{d^{3} q}{2 \omega(q)} \\
& \times \bar{\varphi}\left(q, J \lambda ; \sigma \lambda_{\kappa} \xi\right) \psi(q, J \lambda ; \sigma \lambda \kappa \xi) .
\end{aligned}
$$

Here summation and integration extends over the domain

$$
\left\{(J \lambda ; \sigma \lambda x) \in \vartheta_{n}(J \lambda ; \sigma \lambda x) ; \xi_{1}= \pm 1 ; \xi_{2}= \pm 1\right\} .
$$

We now see that

$$
(\varphi, \psi)=(\stackrel{\bullet}{\varphi}, \dot{\psi})
$$

holds. Hence, in sum, we get the following sequence of isomorphisms:

$$
\mathfrak{W}_{n}(\mathrm{~m}, \mathrm{~s}) \cong \widetilde{\Re}_{n}(\mathrm{~m}, \mathrm{~s}) \cong \AA_{n}(\mathrm{~m}, \mathrm{~s}) \cong \Re_{n}(\mathrm{~m}, \mathrm{~s}) .
$$

We are therefore allowed to identify all these spaces. Because of $(5.29 \mathrm{c}, \mathrm{d})$, we deduce from $(5.36)$ $(U(\Pi) \varphi)\left(q, J \lambda ; \sigma \lambda_{\kappa} \xi\right)$

$(V(T) \varphi)\left(q, J \lambda ; \sigma \lambda_{\kappa} \xi\right)$

$$
=n_{\Pi 1} \xi_{1} D_{\lambda \lambda^{\prime}}^{J}\left(\rho_{\Pi}(q)\right) \varphi\left(\Pi q, J \lambda^{\prime} ; \sigma \lambda_{k} \xi\right),
$$

$$
=n_{T} \xi_{2} D_{\lambda \lambda^{\prime}}^{J}\left(\rho_{\Pi}(q) \epsilon\right) \bar{\varphi}\left(\Pi q, J \lambda^{\prime} ; \sigma \lambda_{\kappa} \xi\right),
$$

which indeed is in agreement with (3.5c) and (3.12), if we make the substitutions

$$
\begin{aligned}
& \eta_{\Pi} \rightarrow n_{\Pi} \xi_{1}, \quad f_{\lambda}(q) \rightarrow \varphi\left(q, J \lambda ; \sigma \lambda_{\mathbf{r}} \xi\right) . \\
& \eta_{T} \rightarrow n_{T} \xi_{2},
\end{aligned}
$$

The subspace of $\mathfrak{\Omega}_{n}(\mathrm{~m}, \mathrm{~s})$ which carries the correct representation of the permutation group we denote by $\Omega_{n}^{\gamma}(m, s)$. Because space inversion and a permutation of the edges of a (three-dimensional) polygon are interchangeable, we get

$$
\pi \Pi x=\Pi \pi x,
$$

and, therefore

$$
B_{x}(\Pi \mathbf{k})=B_{\pi}(\boldsymbol{r}),
$$

if we choose the parametrization $x$ in such a way that $A_{n}(\Pi \mathbf{x})=A_{n}(\boldsymbol{k})$. Hence the space $\AA_{n}^{\gamma}(\mathbf{m}, \mathbf{s})$ is, according to (5.31) and (5.32), characterized as a subspace of $\Omega_{n}(\mathrm{~m}, \mathrm{~s})$ by the following additional properties of $\varphi$ :

$$
\begin{aligned}
& n=2: \eta(\pi)(-1)^{J-2 \bullet} \varphi\left(q, J \lambda ; \lambda_{2} \lambda_{1} \xi\right) \\
&= \varphi\left(q, J \lambda ; \lambda_{1} \lambda_{2} \xi\right) \\
& n \geq 3: B_{*}(\kappa) \varphi(q, J \lambda ; \sigma, \pi \lambda, \pi \kappa \xi) \\
&=
\end{aligned}
$$

If we denote the set in $x$-space on which the function $\varphi$ may be arbitrarily defined [up to the restriction $(\varphi, \varphi)<\infty$ ] by $\vartheta_{n}(x)$, we find

$$
\begin{aligned}
& \vartheta_{n}(q)=\left\{q ; q^{2} \geq|\mathrm{m}|^{2}\right\}, \\
& \vartheta_{n}(J)=\{J ; 2 J \equiv 2|s| \bmod 2\} \\
& \vartheta_{n}(\lambda)=I_{+}(J) .
\end{aligned}
$$

$\vartheta_{n}(\sigma, \lambda)$ belongs to the set of the smallest subsets $\tau$ of $\vartheta_{n}\left(\sigma_{1} \lambda\right)$ with the property

$$
\tau \cup \Pi \tau=\wp_{n}(\sigma, \lambda) .
$$

(Note that the effect of $\Pi$ on a "point in spin-space" is defined by $\Pi(\sigma, \lambda)=(-\sigma,-\lambda)$.)

$\vartheta_{n}(\kappa)$ belongs to the set of the smallest subsets $\tau$ of $\vartheta_{n}(\mathbf{k})$ with the property

$$
\tau \cup \Pi \tau=\dot{\vartheta}_{n}(\mathbf{k})=C_{n}\left(\left[q^{2}\right]^{\mathbf{j}}\right) .
$$

Because of our definition of $\Re_{n}(m, s)$, two functions $\varphi$, which differ only on a set of zero measure in r-space must be considered as the same element of $\Re_{n}(\mathrm{~m}, \mathrm{~s})$. That's why $\vartheta_{n}(\boldsymbol{k})$ is only determined up to a set of zero measure, and, consequently in the case $n \geq 4$, we may assume that $\varphi$ is zero on all points with the property

$$
\Pi \kappa=x
$$

so that $\vartheta_{n}(x)$ contains no such points. In the case $n=3$, all points $x$ have the property $(5.40)$, or, expressed differently,

$$
\vartheta_{3}(\boldsymbol{k})=\stackrel{\vartheta}{3}_{3}(\boldsymbol{k})=C_{3}\left(\left[q^{2}\right]^{1}\right)
$$


holds. In addition, we have

$$
\begin{aligned}
& \vartheta_{n}\left(\xi_{2}\right)=\left\{\xi_{2} ; \xi_{2}= \pm 1 \text { for } n \geq 4 ;\right. \\
& \left.\xi_{2}=1 \quad \text { for } n=2,3\right\} \text {; } \\
& \vartheta_{n}\left(\xi_{1}\right)=\left\{\xi_{1} ; \xi_{1}= \pm 1 \quad \text { for }(\sigma, \lambda) \neq 0\right. \text {; } \\
& \left.\xi_{1}=\xi_{2}(-1)^{J-1,1} \text { for }(\sigma, \lambda)=0\right\} \text {. }
\end{aligned}
$$

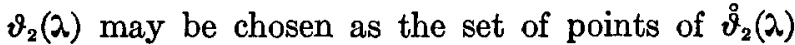
lying on the upper side of the diagonal $\left(\lambda_{1}=-\lambda_{2}\right)$ of the rectangle which is formed by the points of

$$
I_{\tau_{1}}\left(s_{1}\right) \otimes I_{\tau_{2}}\left(s_{2}\right)
$$

and on one half of the diagonal itself [the point $(0,0)$ included, if it belongs to $\vartheta_{2}(\lambda)$, which is exactly the case if $s_{1}$ and $s_{2}$ are integers].

In analogy to the sets $\vartheta_{n}^{\gamma}(x)$ we introduce the sets $\vartheta_{n}^{\gamma}(x) . \vartheta_{2}^{\gamma}(\lambda)$ may be chosen as $\vartheta_{2}(\lambda) \cap \vartheta_{2}^{\gamma}(\lambda)$. ( $\cap$ means intersection), where the last two domains are chosen as indicated above. From (5.36a) and (5.36b) we deduce

$$
\eta(\pi) \varphi\left(q, J \lambda_{;} \lambda_{2} \lambda_{1} \xi\right)=\xi_{1} \varphi\left(q, J \lambda ;-\lambda_{1},-\lambda_{2} \xi\right),
$$

and consequently

$$
\begin{array}{r}
\vartheta_{2}^{\gamma}\left(\xi_{1}\right)=\left\{\xi_{1} ; \xi_{1}= \pm 1 \text { for } \lambda_{1} \neq-\lambda_{2} ;\right. \\
\left.\xi_{1}=\eta(\pi) \text { for } \lambda_{1}=-\lambda_{2}\right\} .
\end{array}
$$

$\vartheta_{n}^{\gamma}(\sigma, \lambda)$ for $n \geq 3$ belongs to the set of the smallest subsets $\tau$ of $\vartheta_{n}(\sigma, \lambda)$ with the property

$$
\bigcup_{g \in \gamma_{n^{\prime}(\text { II })}} g_{\tau}=\gtrless_{n}(\sigma, \lambda),
$$

where $\gamma_{n}^{\prime}(\Pi)$ stands for the group $\gamma_{n}^{\prime}$ supplied by the element $\Pi$ which commutes with the elements $\pi$ of $\gamma_{n}^{\prime}$. Note that the effect of $\pi$ on "a point in spin-

$$
\begin{array}{lll}
\pi \kappa=\kappa, & \pi \in \gamma_{n}^{\prime}, & n=3, \\
g \kappa=\kappa, & g \in \gamma_{n}^{\prime}(I), & n \geq 3
\end{array}
$$

occur in $\vartheta_{n}^{\gamma}(\boldsymbol{x})$.

It is now easy to derive the decomposition rules of the space with respect to the groups $\tilde{P}_{+}^{\dagger}, \tilde{P}$, respectively.

Case $n=2$ : The number $n^{(\gamma)}(M, J)$ of representations $(M, J)$ of the group $\tilde{P}_{+}^{\dagger}$ equals the num-

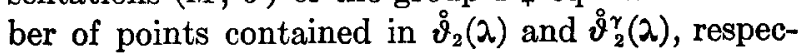
tively, depending on whether the particles are of different kind or not. In formulas,

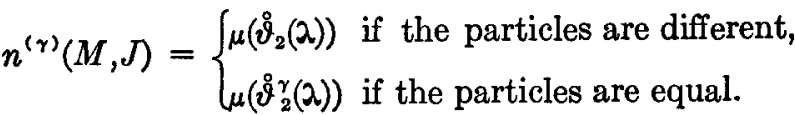

If the particle are different and both have masses different from zero, this number is $\left(s_{1} \geq s_{2}\right)$ :

$$
n(M, J)=\left\{\begin{array}{cr}
\left(2 s_{1}+1\right)\left(2 s_{2}+1\right), & J \geq s_{1}+s_{2}, \\
\left(2 s_{1}+1\right)\left(2 s_{2}+1\right) & \\
-\left(s_{1}+s_{2}-J\right)\left(s_{1}+s_{3}-J+1\right), & s_{1}-s_{2} \leq J \leq s_{1}+s_{2}, \\
(2 J+1)\left(2 s_{2}+1\right), & J \leq s_{1}-s_{2} .
\end{array}\right.
$$

(Compare this with Refs. 3, 5, and 10.)

If it is even (which is always the case unless both of the particles have integral spin) half of it belongs to $\xi_{1}=+1$ and half of it belongs to $\xi_{1}=-1$. In the exceptional case, one of the representations $(M, J)$ belongs to

$$
\xi_{1}=(-1)^{J-a_{1}-c,} \quad \text { (corresponding to } \lambda=0 \text { ), }
$$

and $\frac{1}{2}(n(M, J)-1)$ to $\xi_{1}=+1, \xi_{1}=-1$, respectively. If both particles are equal with mass different from zero, the numbers $n^{\gamma}(M, J)$ are

$$
\begin{array}{ccc} 
& J \geq 2 s & J \leq 2 s \\
\hline \eta(\pi)(-1)^{J-2 s}=+1 & (2 s+1)(s+1) & (J+1)(2 s+1)-\frac{1}{2}[J(J+1)] \\
\eta(\pi)(-1)^{J-2 s}=-1 & (2 s+1) s & 2 J s-\frac{1}{2}[J(J-1)] .
\end{array}
$$

space" is defined by

$$
\pi(\sigma, \lambda)=(\sigma, \pi \lambda) .
$$

Analogous to $\vartheta_{n}^{\gamma}(\sigma, \lambda), \vartheta_{n}^{\gamma}(\boldsymbol{x})$ is defined. By the same reason as in the case of $\vartheta_{n}(\boldsymbol{k}), \vartheta_{n}^{\gamma}(\boldsymbol{k})$ is only determined up to a set of zero measure. That is why we may assume that no point of the submanifolds in $\mathrm{k}$-space defined by
If we denote by $d(J)$ the number of points in $\vartheta_{2}^{\gamma}(\lambda)$ simultaneously lying on the straight line given by the equation $\lambda_{1}=-\lambda_{2}$ we see that

$$
\frac{1}{2}\left(n^{\gamma}(M, J)-d(J)\right)
$$

of the representations $(M, J)$ belongs to $\xi_{1}=+1$ and the same amount belongs to $\xi_{1}=-1$. The rest, namely $d(J)$, belongs to 


$$
\xi_{1}=\eta(\pi) .
$$

The numbers $d(J)$ are given in the following table.

\begin{tabular}{llll}
$2 s$ & $J$ & \multicolumn{1}{c}{$J>2 s$} & \multicolumn{1}{c}{$J \leq 2 s$} \\
\hline even & even & $\frac{1}{2}(2 s+1 \pm 1)$ & $\frac{1}{2}(J+1 \pm 1)$ \\
even & odd & $\frac{1}{2}(2 s+1 \pm 1)$ & $\frac{1}{2}(J \pm 1)$ \\
odd & even & $\frac{1}{2}(2 s+1)$ & $\frac{1}{2} J$ \\
odd & odd & $\frac{1}{2}(2 s+1)$ & $\frac{1}{2}(J+1)$
\end{tabular}

The plus and minus sign in the first two lines simultaneously holds with the corresponding sign in the Eq. (5.33).

If we assume the correct connection between spin and statistics, i.e.,

$$
\eta(\pi)=(-1)^{2 *},
$$

then the numbers of representations $(M, J)$ belonging to $\xi_{1}=+1, \xi_{1}=-1$, respectively, are and we have to complete it by the following statements:

$n \geq 4\left\{\begin{array}{l}\left.1 \text { belongs to } \xi=\left((-1)^{J-1 \star 1}, 1\right)\right\} \quad n=3, \\ 1 \text { belongs to } \xi=\left(-(-1)^{J-1 * 1},-1\right) .\end{array}\right.$

Note especially that

$$
\mu\left(\vartheta_{n}(\sigma, \lambda)\right)=(2 J+1) \prod_{k=1}^{n}\left(2 s_{k}+1\right) .
$$

Now let us consider the space $\mathfrak{S}\left(\vartheta_{n}\right)$ of the functions

$$
f(\sigma, \lambda, \boldsymbol{x})
$$

defined on the domain

$$
\S_{n}(\sigma, \lambda, \mathbf{x}) \text {, }
$$

and assume that $\mathfrak{S}\left(\oiint_{n}\right)$ is equipped with the scalar product

$$
\sum_{\lambda, \sigma} \int d^{3_{n}-6}{ }_{\kappa} \delta\left(q^{2}-M^{2}(\kappa)\right) \bar{f}(\sigma, \lambda, \kappa) g(\sigma, \lambda, \kappa),
$$

\begin{tabular}{cccccc} 
& $\begin{array}{c}2 s \\
J\end{array}$ & $\begin{array}{c}\text { even } \\
\text { even }\end{array}$ & $\begin{array}{c}\text { even } \\
\text { odd }\end{array}$ & $\begin{array}{c}\text { odd } \\
\text { even }\end{array}$ & $\begin{array}{c}\text { odd } \\
\text { odd }\end{array}$ \\
\hline \multirow{3}{*}{$\xi_{1}=+1$} & $J \leq 2 s$ & $(J+1)(s+1)-\frac{J}{2}\left(\frac{J}{2}+1\right)$ & $J s-\frac{(J-1)^{2}}{4}$ & $\frac{1}{2}(2 s+1)(J+1)-\frac{J(J+2)}{4}$ & $J\left(s+\frac{1}{2}\right)-\frac{(J+1)^{2}}{4}$ \\
& $J>2 s$ & $(s+1)^{2}$ & $s(s+1)$ & $\frac{1}{4}(2 s+1)^{2}$ & $s^{2}-\frac{1}{4}$ \\
$\xi_{1}=-1$ & $J \leq 2 s$ & $(J+1) s-\frac{J^{2}}{4}$ & $J s-\frac{J^{2}-1}{4}$ & $\frac{1}{2}(2 s+1)(J+1)-\frac{J^{2}}{4}$ & $J\left(s+\frac{1}{2}\right)-\frac{J^{2}-1}{4}$ \\
& $J>2 s$ & $s(s+1)$ & $s^{2}$ & $\frac{1}{4}(2 s+1)(2 s+3)$ & $\left(s+\frac{1}{2}\right)^{2}$
\end{tabular}

For $n \geq 3$, to each point $\kappa \in \vartheta_{n}^{(\gamma)}(\kappa)$ there exists

$$
\begin{aligned}
\mu_{n}^{(\gamma)}, & n=3, \\
2 \mu_{n}^{(\gamma)}, & n \geq 4
\end{aligned}
$$

representations $(M, J)$ of $\tilde{P}_{+}^{\dagger}$, where

$$
\mu_{n}^{(\gamma)}=\mu\left(\vartheta_{n}^{(\gamma)}(\sigma, \lambda)\right) \text {. }
$$

If not all of the numbers $s_{k}$ are integral,

$n \geq 4\left\{\begin{array}{lll}\frac{1}{2} \mu_{n}^{(\gamma)} & \text { belongs to } & \xi=(1,1) \\ \frac{1}{2} \mu_{n}^{(\gamma)} & \text { belongs to } & \xi=(-1,1) \\ \frac{1}{2} \mu_{n}^{(\gamma)} & \text { belongs to } & \xi=(1,-1), \\ \frac{1}{2} \mu_{n}^{(\gamma)} & \text { belongs to } \xi=(-1,1) .\end{array}\right\}$

If all the numbers $s_{k}$ are integers, $\mu_{n}^{(\gamma)}$ in the table above has to be replaced by

$$
\mu_{n}^{(\gamma)}-1 \text {, }
$$

where the summation and integration are extended over the domain (5.48). We also consider that subspace $\mathfrak{S}\left(\wp_{n}^{\gamma}\right)$ of $\mathfrak{S}\left(\wp_{n}\right)$ whose elements satisfy the relation

$$
\begin{aligned}
B_{\pi}(\boldsymbol{\kappa}) f(\sigma, \pi \lambda, \pi \kappa) & =\eta(\pi) f(\sigma, \lambda, \boldsymbol{\kappa}), & & n \geq 3, \\
\eta(\pi)(-1)^{J-2 s} f(\pi \lambda) & =f(\lambda), & & u=2 .
\end{aligned}
$$

Let us denote by

$$
\left\langle\mathbf{v}\left|H^{(\gamma)}(\mathrm{m}, \mathrm{s}, J M)\right| \sigma, \lambda, \mathrm{k}\right\rangle
$$

$n=3$, a complete orthonormal system of functions of the space $\mathfrak{E}\left(\hat{\vartheta}_{n}^{(\gamma)}\right)$ which may depend also on the variables $\mathrm{m}, \mathrm{s}, M, J$. In the future we shall suppress this dependence to make the formulas look less cumbersome. Completeness and orthogonality are expressed by the relations

$$
\begin{array}{r}
\sum_{2 \sigma} \int d^{3_{n}-\sigma} \kappa \delta\left(q^{2}-M^{2}(\boldsymbol{\kappa}) \overline{\left\langle v\left|H^{(\gamma)}\right| \sigma, \lambda, \kappa\right\rangle}\right. \\
\times\left\langle v^{\prime}\left|H^{(\gamma)}\right| \sigma, \lambda, \kappa\right\rangle=\delta_{v^{\prime}},
\end{array}
$$




$$
\begin{aligned}
& \sum_{\gamma} \delta\left(q^{2}-M^{2}(\boldsymbol{k})\right)\langle\boldsymbol{v}| \overline{H^{(\gamma)}|\sigma, \lambda, \boldsymbol{k}\rangle} \\
& \times\left\langle\boldsymbol{v}\left|H^{(\gamma)}\right| \sigma^{\prime}, \lambda^{\prime}, \boldsymbol{\kappa}^{\prime}\right\rangle=\delta^{3 n-6}\left(\boldsymbol{\kappa}-\boldsymbol{k}^{\prime}\right) \delta_{2 \lambda^{\prime}} \delta_{\sigma \sigma^{\prime}}
\end{aligned}
$$

In (5.49) the parameters $v$ "counting the members of the system" may partially be discreet, partially continuous. Accordingly we have to replace the sum in $(5.50 \mathrm{~b})$ partially by an integral and the Kroneckersymbol on the right-hand side of (5.50a) by $\delta$-functions. If we start from $\AA_{\mathrm{a}}(\mathrm{m}, \mathrm{s})$ and perform the transformation

$$
\begin{aligned}
\hat{\varphi}(q ; J \lambda ; v) & =\sum_{\lambda \sigma} \int d^{3 n-\sigma} \delta\left(q^{2}-M^{2}(\boldsymbol{k})\right) \\
& \times\langle\mu|H| \sigma, \lambda, \boldsymbol{\kappa}\rangle \dot{\varphi}_{\lambda}(q ; J \lambda \sigma ; \boldsymbol{\kappa}),
\end{aligned}
$$

where summation and integration again extend over the domain (5.48), then the space $\hat{\Omega}_{n}(\mathrm{~m}, \mathrm{~s})$ of the functions $\hat{\varphi}$ equipped with the scalar product

$$
(\hat{\varphi}, \hat{\psi})=\sum_{J, \lambda} \sum_{v} \int d^{4} q \overline{\hat{\varphi}}(q ; J \lambda ; v) \hat{\psi}(q ; J \lambda ; v)
$$

is operator-isomorphic to the original one with respect to the group $\tilde{P}_{+}^{\dagger}$ and therefore splits into irreducible spaces with character $(M, J)$ in the same way as $\mathfrak{R}_{n}(\mathrm{~m}, \mathrm{~s})$.

It is easily seen that (5.51) is the most general transformation with this property. The most general $\mathrm{C}-\mathrm{G}$ coefficient of the group $\tilde{P}_{+}^{\dagger}$ is therefore defined by

$$
\begin{aligned}
& \hat{\varphi}(q ; J \lambda ; v) \\
& \quad=\sum_{2} \int \frac{1}{2^{n}} \frac{d^{3 n} p}{\omega_{1}\left(\mathbf{p}_{1}\right) \cdots \omega_{n}\left(\mathbf{p}_{n}\right)}\left(\begin{array}{c|c}
M J & \mathbf{m s} \\
q \lambda & \mathbf{p} 2
\end{array}\right)_{(\gamma)} f_{2}(\mathbf{p})
\end{aligned}
$$

and is consequently given by the expression

$$
\begin{gathered}
\left(\begin{array}{c|c}
M J & \mathrm{~ms} \\
q \lambda & \mathrm{p} \lambda
\end{array}\right)=\delta^{4}\left(q-q^{\prime}\right)\left[(2 J+1) A_{n}^{-1}(\mathbf{k})\right]^{\frac{1}{2}} \\
\times \sum_{\lambda^{\prime}, \sigma} \mathfrak{D}_{\lambda^{\prime} / 2}^{\mathrm{s}}(\mathrm{p}) D_{\lambda_{\sigma}}^{J}(\rho)\left\langle v\left|H^{(\gamma)}\right| \lambda^{\prime}, \sigma, \boldsymbol{\kappa}\right\rangle .
\end{gathered}
$$

Here $q^{\prime}, \rho$, and $x$ have the values determined by $\mathbf{p}$ through the relation (4.21). In an analogous way we determine the $\mathrm{C}-\mathrm{G}$ coefficients of $\bar{P}$ considering only representations for which

$V^{2}(T)=(-1)^{2 s}$ and $V^{2}(\Pi T)=(-1)^{2 s}$ (if $\eta_{\Pi}$ is real)

We only have to replace the functions

$$
\left\langle\boldsymbol{v}|H(\gamma)| \lambda^{\prime}, \sigma, \mathbf{k}\right\rangle
$$

in (5.54) by the following linear combinations of these functions:

$$
\begin{aligned}
& \left\langle v\left|H^{(\gamma)}(\xi)\right| \sigma \lambda \mathbf{x}\right\rangle \\
& =\frac{1}{4}\left\{\left[\left\langle v\left|H^{(\gamma)}\right| \sigma, \lambda, \kappa\right\rangle+\xi_{2}\left\langle v\left|H^{(\gamma)}\right| \sigma, \lambda, \Pi \kappa\right\rangle\right]\right. \\
& +\xi_{1} \xi_{2}(-1)^{|\rho|-|\lambda|-J+\sigma}\left[\left\langle v\left|H^{(\gamma)}\right|-\sigma,-\lambda, \boldsymbol{k}\right\rangle\right. \\
& \left.\left.+\xi_{2}\left\langle v\left|H^{(\gamma)}\right|-\sigma,-\lambda, \Pi \kappa\right\rangle\right]\right\} \text {. }
\end{aligned}
$$

If we make the identification of all the spaces isomorphic to each other [see (5.38)], the transformations which reduce the space $\bigvee_{n}(\mathrm{~m}, \mathrm{~s})$ are seen to be orthogonal. That's why we know without direct calculation that the $\mathrm{C}-\mathrm{G}$ coefficients satisfy the relations

$$
\begin{array}{r}
\sum_{J} \sum_{\gamma, \lambda} \int d^{4} q \overline{\left(\begin{array}{c|c|c}
M J & \mathrm{~ms} \\
v \lambda & \mathbf{p}^{(1)} \lambda^{(1)}
\end{array}\right)}\left(\begin{array}{c}
M J \\
v i \lambda
\end{array}\right. \\
=2^{n} \prod_{k=1}^{n} \omega\left(p_{k}^{(1)}\right) \delta^{3}\left(\mathfrak{p}_{k}^{(1)}-\mathfrak{p}_{k}^{(2)}\right),
\end{array}
$$

$\sum_{2} \int \frac{1}{2^{n}} \frac{d^{3 n} p}{\omega_{1}\left(\mathfrak{p}_{1}\right) \cdots \omega_{n}\left(\mathfrak{p}_{n}\right)}$

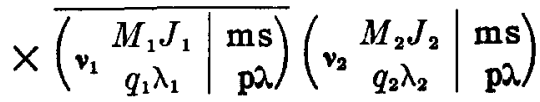

$$
\begin{aligned}
& =\delta_{\nu_{1} \nu_{z}} \delta^{4}\left(q_{1}-q_{2}\right) \cdot \delta_{i_{1} j_{2}} \cdot \delta_{\lambda_{1} \lambda_{2}} .
\end{aligned}
$$

The analogous relations are satisfied by the $\mathrm{C}-\mathrm{G}$ coefficients of $\tilde{P}$.

\section{THE PHYSICAL MEANING OF THE QUANTITIES ON WHICH THE FUNCTIONS $\because$ DEPEND.}

In this section, we want to find the physical meaning of the quantities $\lambda$ and $\sigma$ on which the functions $\vartheta_{\lambda}(q ; j \lambda \sigma ; \boldsymbol{x})$ depend. For this purpose we consider in $\mathfrak{S}_{n}(\mathrm{~m}, \mathrm{~s})$ the representation of $\tilde{P}_{+}^{\dagger}$ which is isomorphic to that one taking place in $\mathfrak{S}\left(m_{l}, s_{l}\right)$, namely

$$
\begin{aligned}
\left(U_{l}(a) f\right)(\mathbf{p}) & =e^{i p l a} f(\mathbf{p}), \\
\left(U_{l}(A) f\right)(\mathbf{p}) & =D^{\prime l}\left(\rho_{l}\left(p_{l}, A\right)\right) f\left(\Lambda^{-1}(A)_{l} \mathbf{p}\right),
\end{aligned}
$$

where $\Lambda^{-1}(A)_{l} \mathrm{p}$ is defined by

$$
\Lambda^{-1}(A)_{l} \mathrm{P}=\left(p_{1} \cdots p_{l-1}, \Lambda^{-1}(A) p_{l}, p_{l+1} \cdots p_{n}\right) \text {. }
$$

It follows immediately

$$
U(A)=\prod_{l=1}^{n} U_{l}(A) .
$$

The infinitesimal translations in the representation $U_{l}$ we call $P_{l}$.

Now let

$$
v\left(p_{l}\right)=\alpha_{l}\left(p_{l}\right) v \alpha^{-1}\left(p_{l}\right)
$$

be an element of the little group of $p_{\sim l}$. 
It follows

$$
\rho\left(p_{l}, v\left(p_{l}\right)\right)=v
$$

and therefore

$$
\left(U_{l}\left(v\left(p_{l}\right)\right) F^{\prime}\right)(\mathbf{p})=D^{\prime \prime}\left(r_{l}(\mathbf{p}) v r_{l}^{-1}(\mathbf{p})\right) F(\mathbf{p}),
$$

especially

$$
\begin{aligned}
\left(U_{l}\left(\alpha_{l}\left(p_{l}\right) r_{l}^{-1}(\mathbf{p}) \rho(\chi) r_{l}(\mathbf{p}) \alpha^{-1}\left(p_{l}\right)\right) F\right)(\mathbf{p}) & \\
& =D^{\prime \prime}(\rho(\chi)) F(\mathbf{p}) .
\end{aligned}
$$

If we go to infinitesimal transformations $\rho(\chi)$, we get from the last equation

$$
W_{\gamma}^{(l)} \Lambda_{3}^{\gamma}\left(r_{l}(\mathrm{p}) \alpha^{-1}\left(p_{l}\right)\right) F(\mathrm{p})=\lambda_{l} F(\mathrm{p}),
$$

where $W_{\gamma}^{(l)}$ is the operator defined in (3.19) with the difference that $\mathfrak{I}$ and $\mathfrak{N}$ have to be replaced by $\mathfrak{F}^{(l)}$ and $\mathfrak{\Re}^{(l)}$, the infinitesimal Lorentz transformations in the representation $U_{l}$ of $\tilde{P}_{+}^{\dagger}$.

From (6.3) we deduce immediately: The functions

$$
\dot{\varphi}_{\lambda}(q ; J \lambda \sigma ; \boldsymbol{\kappa})
$$

are eigenfunctions of the operator

$$
W_{\gamma}^{(l)} \Lambda_{3}^{\gamma}\left(r_{l}(\mathbf{P}) \alpha_{l}^{-1}\left(P_{l}\right)\right)
$$

to the eigenvalue $\lambda_{l}$. Here the replacement

$$
p_{k} \rightarrow P_{k}
$$

in the expression $r_{l}(p) \alpha_{l}^{-1}\left(p_{l}\right)$ is defined by corresponding power series. No difficulties arise from the indefiniteness of the order of the different operators because the $P_{l}$ commute. In general the operator (4) depends on all the momenta operators $P_{1} \cdots P_{n}$.

However, if we make the WJW choice

$$
\alpha^{u}\left(b_{l}\right)=\alpha\left[b_{l}\right],
$$

and if we confine ourselves to the center-of-mass system, this operator becomes very simple. In this case we get

$$
\alpha_{l}\left(p_{l}\right) r_{l}^{-1}(\mathbf{p})=\rho \alpha\left[b_{l}\right]=\alpha\left[p_{l}\right] \rho(\chi(\mathbf{p})),
$$

where $\chi(\mathbf{p})$ in general depends on all the momenta $p_{k}$. But if we introduce this expression in (6.2), the $\rho(\chi(\mathbf{p}))$ drops out and we get

$$
\left(U_{l}\left(\alpha\left[p_{l}\right] \rho(\chi) \alpha^{-1}\left[p_{l}\right] F\right)(\mathbf{p})=\mathfrak{D}^{s i}(\rho(\chi)) F(\mathbf{p}),\right.
$$

i.e., $\wp_{2}\left(q_{k} ; j \lambda \sigma ; \boldsymbol{\kappa}\right)$ [where $q_{k}=(M, 0)$ ] is eigenfunction to the value $\lambda_{l}$ of the operator

$$
W_{\gamma}^{(l)} \Lambda_{3}^{\gamma}\left(\alpha^{-1}\left[P_{l}\right]\right),
$$

which is the helicity operator as we have seen in Sec. 3. Now let's start from the equation
$\left(U\left(\rho_{0} \rho(\chi) \rho_{0}^{-1}\right) \tilde{\varphi}\right)\left(q_{k}, \rho_{0} \rho\left(\chi^{\prime}\right), \kappa\right)=\tilde{\varphi}\left(q_{k}, \rho_{0} \rho\left(\chi^{\prime}+\chi\right), \kappa\right)$.

Here

$$
\rho_{0}=\rho \rho\left(-\chi^{\prime}\right) \equiv \rho_{0}(p) \text {, }
$$

where $\rho$ is determined by $p$ through the relation (4.21). If we set by definition

$$
\tilde{\varphi}\left(q_{k}, \rho_{0}, \sigma, \kappa\right)=\frac{1}{4 \pi} \int_{0}^{4 \pi} d \chi^{\prime} \tilde{\varphi}\left(q_{k}, \rho_{0} \rho\left(\chi^{\prime}\right), \kappa\right),
$$

we get

$$
\left(U\left(\rho_{0} \rho(\chi) \rho_{0}^{-1}\right) \tilde{\varphi}\right)\left(q_{k}, \rho_{0}, \sigma, \boldsymbol{\kappa}\right)=e^{i \sigma \chi} \tilde{\varphi}\left(q_{k}, \rho_{0}, \sigma, \boldsymbol{\kappa}\right),
$$

or, if we go to infinitesimal transformations $\rho(\chi)$,

$$
\mathfrak{I n}(\mathbf{p}) \tilde{\varphi}\left(q_{k}, \rho_{0}, \sigma, \boldsymbol{k}\right)=\sigma \tilde{\varphi}\left(q_{k}, \rho_{0}, \sigma, \boldsymbol{k}\right) .
$$

Here $n(\mathbf{p})$ is the vector we get if we apply the rotation

$$
\Lambda\left(\rho_{0}^{-1}(\mathrm{p})\right)
$$

to the unit vector pointing in the direction of the positive 3 -axis. If we again replace the momentum $p_{l}$ in $n(\mathrm{p})$ by the corresponding operator in the representation $U_{l}$, we may complete the transformation (5.23) in (6.5) without difficulties. We then observe that $\sigma$ in $\vartheta(q ; j \lambda \sigma ; \boldsymbol{k})$ has the meaning of the total angular momentum in the center-of-mass system relative to an axis which is connected with the momenta of the $n$ particles in a definite way. The situation is similar to the case of a top: $\sigma$ corresponds to the angular momentum relative to the figure axis and $\lambda$ to the one relative to an axis fixed in space.

\section{APPLICATION TO THE S-MATRIX}

We consider the $S$-matrix of a process

$$
\begin{aligned}
a_{1}^{(1)}+a_{2}^{(1)}+ & \cdots+a_{n_{1}}^{(1)} \\
& \rightarrow a_{1}^{(2)}+a_{2}^{(2)}+\cdots+a_{n_{1}}^{(2)} .
\end{aligned}
$$

Let $m_{k}^{(i)}$ be the mass, $s_{k}^{(i)}$ the spin of the particle, $a_{k}^{(i)} p_{k}^{(i)}$ its momentum, and $\lambda_{k}^{(i)}$ the third component of its spin (for definition of this quantity in a coordinate-system in which the momentum $p_{k}^{(i)}$ is measured, see Sec. 3). The $S$-matrix maps the $\Pi_{k=1}^{n_{1}}\left(2 s_{k}^{(1)}+1\right)$-dimensional spin space of the incoming particles on the $\Pi_{k=1}^{n=}\left(2 s_{k}^{(2)}+1\right)$-dimensional spin space of the outgoing particles, and is, at the same time, a Lorentz-invariant generalized function of the variables

$$
\mathbf{p}^{(i)}=\left(p_{1}^{(i)} \cdots p_{n i}^{(i)}\right)(i=1,2): S\left(\mathbf{p}^{(1)}, \mathbf{p}^{(2)}\right) .
$$

From translation invariance, one derives that the support of $S$ is the submanifold on $V^{+}\left(\mathrm{m}^{(1)}\right) \otimes$ 
$V^{+}\left(\mathrm{m}^{(2)}\right)$ defined by

$$
q^{(1)}=q^{(2)} ; \quad q^{(i)}=\sum_{k=1}^{n_{i}} p_{k}^{(i)} .
$$

Lorentz invariance implies

$$
\begin{aligned}
\mathbb{D}^{8(1)}\left(\rho\left(\mathbf{p}^{(1)}, A\right)\right) & S\left(\Lambda^{-1}(A) \mathbf{p}^{(1)}, \Lambda^{-1}(A) \mathbf{p}^{(2)}\right) \\
& \times \mathbb{D}^{\mathbf{s}^{(2)}}\left(\rho^{\dagger}\left(\mathbf{p}^{(2)}, A\right)\right)=S\left(\mathbf{p}^{(1)}, \mathbf{p}^{(2)}\right) .
\end{aligned}
$$

If we now define

$$
\begin{aligned}
T\left(\mathbf{p}^{(1)}, \mathrm{p}^{(2)}\right) & \delta^{4}\left(q^{(1)}-q^{(2)}\right) \\
= & \mathbb{D}^{\mathbf{s}^{(1)}}\left(\mathbf{p}^{(1)}\right) S\left(\mathbf{p}^{(1)} \mathbf{p}^{(2)}\right) \mathbb{D}^{\mathbf{s}^{(3) t}}\left(\mathbf{p}^{(2)}\right),
\end{aligned}
$$

where we subtract a $\delta^{4}\left(q^{(1)}-q^{(2)}\right)$ function on the right side, if the process under consideration is an elastic scattering, Lorentz invariance reads

$$
T\left(\mathbf{p}^{(1)}, \mathbf{p}^{(2)}\right)=T\left(\Lambda^{-1}(A) \mathbf{p}^{(1)}, \Lambda^{-1}(A) \mathbf{p}^{(2)}\right) .
$$

Hence the inverse of (2) is

$$
S\left(\mathbf{p}^{(1)}, \mathbf{p}^{(2)}\right)=\delta^{4}\left(q^{(1)}-q^{(2)}\right) \hat{T}\left(\mathbf{p}^{(1)}, \mathbf{p}^{(2)}\right),
$$

where

$\hat{T}\left(\mathbf{p}^{(1)}, \mathbf{p}^{(2)}\right)=\mathbb{D}^{\mathbf{s}^{(1)} \dagger}\left(\mathbf{p}^{(1)}\right) T\left(\mathbf{p}^{(1)}, \mathbf{p}^{(2)}\right) \mathbb{D}^{\mathrm{s}(1)}\left(\mathbf{p}^{(2)}\right)$

gives a representation of the $S$-matrix as a sum of the covariants of $\tilde{P}_{+}^{\dagger}$, each multiplied by an invariant amplitude $T\left(\mathbf{p}^{(1)}, \mathbf{p}^{(2)}\right)$.

Because of $q-q^{(1)}=q^{(2)}$, we may write, according to (5.16),

$$
\begin{aligned}
T\left(\mathrm{p}^{(1)}, \mathrm{p}^{(2)}\right)=\tau_{1}\left(q ; \rho^{(1)}, \rho^{(2)} ;\right. & \left.\boldsymbol{\kappa}^{(1)} \boldsymbol{\kappa}^{(2)}\right) \\
& \times A_{n_{1}}^{-\frac{1}{3}}\left(\boldsymbol{\kappa}^{(1)}\right) A_{n_{2}}^{-\frac{1}{2}}\left(\boldsymbol{\kappa}^{(2)}\right) .
\end{aligned}
$$

(From the rigorous mathematical point of view, this transformation would need a justification depending on the test-function space, which is used to define the general functions.) Lorentz invariance now reads

$$
\begin{aligned}
\tau_{1}\left(\Lambda^{-1}(A) q ; \rho^{-1}(q, A) \rho^{(1)}\right. & \left., \rho^{-1}(q, A) \rho^{(2)} ; \kappa^{(1)} \kappa^{(2)}\right) \\
& =\tau_{1}\left(q ; \rho^{(1)}, \rho^{(2)} ; \kappa^{(1)}, \kappa^{(2)}\right) .
\end{aligned}
$$

Choosing

$$
A=\alpha(q) \rho^{(2)},
$$

we get

$$
\rho(q, A)=\rho^{(2)}, \quad \Lambda^{-1}(A) q=(M, 0)=q_{k},
$$

and therefore

$$
\begin{aligned}
\tau_{1}\left(q ; \rho^{(1)}, \rho^{(2)} ; \boldsymbol{\kappa}^{(1)}, \boldsymbol{\kappa}^{(2)}\right) \\
\quad=\tau_{1}\left(q_{k} ; \rho, \mathbb{1} ; \boldsymbol{\kappa}^{(1)}, \boldsymbol{\kappa}^{(2)}\right) \equiv \tau\left(M, \rho ; \boldsymbol{\kappa}^{(1)} \boldsymbol{\kappa}^{(2)}\right),
\end{aligned}
$$

where $\rho=\left(\rho^{(2)}\right)^{-1} \rho^{(1)}$ and the sign $\equiv$ means "equal per definition". It is clear that

$$
M\left(\boldsymbol{\kappa}^{(1)}\right):=M\left(\boldsymbol{\kappa}^{(2)}\right)=M=\left(q^{2}\right)^{2} .
$$

Performing the transformation

$$
\begin{aligned}
& \tau\left(M J ; \sigma_{1} \kappa^{(1)} ; \sigma_{2} \boldsymbol{\kappa}^{(2)}\right) \\
& =(2 J+1)^{\frac{1}{2}} \int d \rho D_{\sigma_{3} \sigma_{2}}^{J}(\rho) \tilde{\tau}\left(M, \rho ; \boldsymbol{\kappa}^{(1)} \kappa^{(2)}\right),
\end{aligned}
$$

we finally get "the generalized partial wave decomposition"

$$
\begin{aligned}
& T\left(\mathbf{p}^{(1)} \mathbf{p}^{(2)}\right)=A_{n_{1}}^{-\frac{1}{2}}\left(\boldsymbol{\kappa}^{(1)}\right) A_{n_{3}}^{-\frac{1}{2}}\left(\boldsymbol{\kappa}^{(2)}\right) \\
& \times \sum_{J \sigma_{1} \sigma_{3}}(2 J+1)^{\frac{1}{2}} \tau\left(M J ; \sigma_{1} \boldsymbol{k}^{(1)}, \sigma_{2} \kappa^{(2)}\right) \bar{D}_{\sigma_{2} \sigma_{1}}^{J}(\rho) .
\end{aligned}
$$

In the case $n_{1}=n_{2}=2$, the substitution

$$
\begin{aligned}
& \rho_{1} \rightarrow \rho_{1} \rho\left(\chi_{1}\right), \\
& \rho_{2} \rightarrow \rho_{2} \rho\left(\chi_{2}\right),
\end{aligned}
$$

which leads to

$$
\rho \rightarrow \rho\left(-\chi_{2}\right) \rho \rho\left(\chi_{1}\right)
$$

must leave all the equations invariant. It is easily seen that this leads to the relations

$$
\begin{aligned}
& \lambda_{1}^{(1)}-\lambda_{2}^{(1)}=\sigma_{1}, \\
& \lambda_{1}^{(2)}-\lambda_{2}^{(2)}=\sigma_{2} ;
\end{aligned}
$$

if $n_{1}=2, n_{2} \geq 3$, only the first of these relations is valid, if $n_{1} \geq 3, n_{2}=2$, only the second one. In any case (also in the case $n_{1} \geq 3, n_{2} \geq 3$ ), the summation in (7.5) has to be carried out over those $J$, for which

$$
2 J \equiv 2\left|s^{(1)}\right| \equiv 2\left|s^{(2)}\right| \bmod 2 .
$$

According to $(5.29 \mathrm{c})$, invariance with respect to space inversion has the consequence

$$
\begin{aligned}
& \tau_{\lambda^{(1) 2(3)}}\left(M J ; \sigma_{1} \kappa^{(1)}, \sigma_{2} \kappa^{(2)}\right) \\
& =\eta(\Pi) \tau_{-2(1),-2(1)}\left(M, J ;-\sigma_{1} \Pi k^{(1)},-\sigma_{2} \Pi \kappa^{(2)}\right),
\end{aligned}
$$

where

$$
\eta(I I)=\mathbf{n}_{\mathrm{II}}^{(1)} \bar{n}_{I I}^{(2)}(-1)^{|\varepsilon(1)|-|\lambda(1)|+|\varepsilon(s)|-|\lambda(1)|-2 J+\sigma_{1}+\sigma \theta} .
$$

All the results of this section can also be derived using the fact that, according to group-theoretical considerations, the $S$-matrix, being an operator-isomorphism with respect to the group $\tilde{P}_{+}^{\uparrow}$ which maps $\mathfrak{W}_{n_{1}}\left(\mathrm{~m}^{(1)}, \mathrm{s}^{(1)}\right)$ onto $\mathfrak{E}_{n_{\mathrm{g}}}\left(\mathrm{m}^{(2)}, \mathrm{s}^{(2)}\right)$, must have the representation

$$
\begin{array}{r}
\int d^{4} q \sum_{J, \lambda} \sum_{v_{2}, v_{2}} \overline{\left(\begin{array}{c|c|c}
M J & \mathrm{~m}^{(1)} \mathrm{s}^{(1)} \\
v_{1} & q \lambda & \mathrm{p}^{(1)} \lambda^{(1)}
\end{array}\right)}\left(\begin{array}{c}
M J \\
v_{2} \\
q \lambda
\end{array} \mid \begin{array}{c}
\mathrm{m}^{(2)} \mathrm{s}^{(2)} \\
\mathrm{p}^{(2)} \lambda^{(2)}
\end{array}\right) \\
\times\left\langle v_{1}|\tau(J M)| v_{2}\right\rangle,
\end{array}
$$


where the $\mathrm{C}-\mathrm{G}$ coefficients

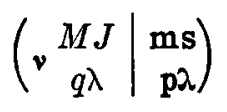

are defined in (5.54).

Indeed if we set

$$
\begin{array}{r}
\tau_{\left.\lambda^{(1)}\right)_{(2)}}\left(M J ; \sigma_{1} \boldsymbol{k}^{(1)}, \sigma_{2} \mathbf{k}^{(2)}\right)=\sum_{v_{1}, v,} \overline{\left\langle v_{1}|H| \sigma_{1} \lambda^{(1)}, \boldsymbol{k}^{(1)}\right\rangle} \\
\times\left\langle v_{1}|\tau(J M)| v_{2}\right\rangle\left\langle v_{2}|H| \sigma_{2} \lambda^{(2)}, \boldsymbol{x}^{(2)}\right\rangle
\end{array}
$$

(7.6) is in complete agreement with the relation we obtain if we combine (7.5) and (7.2).

In an analogous way, we may define a decomposition of the $S$-matrix with respect to the group $\tilde{P}$ using the corresponding $\mathrm{C}-\mathrm{G}$ coefficients given in (5.54) and (5.55).

Finally, we remark that, according to (3.23) in the case $m_{k}^{(i)}>0$, the connection between our $\hat{T}\left(\mathbf{p}^{(1)}, \mathbf{p}^{(2)}\right)$-matrices and the $\sum_{k, i} \oplus\left(s_{k}^{(i)}, 0\right)$ tensors (with respect to $L_{+}^{\dagger}$ ) of $\operatorname{Stapp}^{11}$ and Hepp ${ }^{12}$ is given by

$$
\begin{aligned}
& \mathbb{D}^{s}\left(\alpha\left(\mathbf{p}^{(1)}\right)\right) \hat{T}\left(\mathbf{p}^{(1)}, \mathbf{p}^{(2)}\right) \mathbb{D}^{\mathbf{s}}\left(\epsilon \alpha^{T}\left(\mathbf{p}^{(2)}\right)\right)=M\left(\mathbf{p}^{(1)}, \mathbf{p}^{(2)}\right) \\
& {\left[M\left(\mathbf{p}^{(1)}, \mathbf{p}^{(2)}\right)=\sum_{k, i} \oplus\left(s_{k}^{(i)}, 0\right) \text {-tensor }\right] .}
\end{aligned}
$$

Notes added before print:

A. After having finished the preprint of this paper it was learned by the author that two other authors have written a paper on a similar subject: P. Moussa and R. Stora, "Some Remarks on the Product of Irreducible Representations of the Inhomogeneous Lorentz Group (Preprint, edited at Centre d'Etudes Nucléaire de Saclay, Gif-sur-Yvettes; France.)

R. Stora has called to this author's attention their paper and their method of reducing the product representation applied in their work: the method of induced representations, presented in Mackey's book. [G. W. Mackey, The Theory of GroupRepresentations (The University of Chicago Press, Chicago, 1955)] It turned out that the method applied in the present paper is strongly related to Mackey's general scheme.

I wish to thank Dr. Stora for the correspondence concerning this point.

B. A reader looking through the present paper without studying it in detail might get the impression that, besides the helicity coupling scheme, no other scheme has been treated, which wouldn't justify the term "most general" in the title. That's why the author wants to emphasize that the $\mathrm{C}-\mathrm{G}$ coefficients
(5.54) are quite general and especially that it includes also the $l-s$ coupling scheme of $\mathrm{Joos}^{5}$ and Macfarlane. ${ }^{1}$

To show this in some detail, assume $m_{i}>0$ $(i=1 \cdots n)$ and replace $(4.44)$ by

$$
\alpha_{l}^{u}\left(b_{l}\right)=\alpha\left\{b_{l}\right\} \text {. }
$$

Then (5.54) is formally unchanged, the only difference being that $D_{\lambda^{\prime} \lambda}^{i}(p)$ is defined with the help of

$$
r_{l}\{\mathbf{p}\}=\alpha^{-1}\left\{b_{l}\right\} a^{-1}(\mathbf{p}) \alpha_{l}\left(p_{l}\right)
$$

instead of

$$
r_{l}[\mathbf{p}]=\alpha^{-1}\left[b_{l}\right] a^{-1}(\mathbf{p}) \alpha_{l}\left(p_{l}\right) .
$$

In addition, we choose $\left\langle\boldsymbol{v}|H| \lambda^{\prime}, \sigma, \boldsymbol{x}\right\rangle$ in (5.54) to be

$$
\left(a_{\lambda}^{s} \tau|\tau| s\right), \quad v=(l, \tau, \zeta)
$$

i.e., the C-G coefficient of $S U(2)$ which composes $s_{1} \cdots s_{n}, l$ to $J . \tau=\sigma-\sum_{i-1}^{n} \lambda^{\prime} ; \zeta$ is conveniently chosen to be $\zeta=\left(s_{12}, s_{123} \ldots s_{12} \ldots n\right)$, where $s_{12 \ldots k}$ is obtained by composing $s_{1} \cdots s_{k}$. Because of the relation

$$
r_{l}\{\mathbf{p}\}=\rho\left[b_{l}\right] r_{l}[\mathbf{p}]
$$

following from (B2), (B3), and (2.21), the procedure described so far is equivalent to directly choosing

$$
\left\langle\boldsymbol{v}|H| \lambda^{\prime}, \sigma, \boldsymbol{x}\right\rangle
$$

in (5.54) to be

$$
\begin{aligned}
& \sum_{\lambda^{\prime \prime}} D_{\lambda^{\prime} \cdot \lambda^{\prime}}^{s}\left(\rho[(b])\left(\left.z_{\lambda^{\prime} \prime^{\prime}}^{z}\right|_{\sigma} ^{J} s\right) .\right. \\
& \sum_{i=1}^{n} \lambda_{i} \cdots+i=0
\end{aligned}
$$

It is easy to see that this choice leads to the C-G coefficients of $\mathrm{Joos}^{5}$ and Macfarlane ${ }^{1}$ in the case $n=2$. Notice that the choice (1) implies $y\left(b_{1}, \chi\right)=$ $y\left(b_{2}, \chi\right)=\rho(-\chi)$ [Compare (4.40)], such that in the case $n=2: \lambda_{1}+\lambda_{2}=\sigma$ and therefore $\tau=0$. For $n \geq 3$, however, it leads to a simpler expression than the one obtained by Macfarlane who essentially constructs the C-G coefficients for $n \geq 3$ according to the "Dalitz-scheme" (1), (2) $\rightarrow(12) ;(12)(3) \rightarrow$ (123), etc. using only $\mathrm{C}-\mathrm{G}$ coefficients for $n=2$.

\section{ACKNOWLEDGMENTS}

I wish to thank Professor Williamson for the critical study of this paper. Also, some clarifying discussions with Dr. K. Hepp and S. Albeverio at an earlier stage of this work are gratefully acknowledged. 